Article

\title{
Study of Allelopathic Interaction of Essential Oils from Medicinal and Aromatic Plants on Seed Germination and Seedling Growth of Lettuce
}

\author{
Somayeh Mirmostafaee ${ }^{1}$, Majid Azizi ${ }^{1, *(1)}$ and Yoshiharu Fujii ${ }^{2, *(1)}$ \\ 1 Department of Horticulture and Landscape Engineering, Faculty of Agriculture, Ferdowsi University of \\ Mashhad, Mashhad 9177948974, Iran; smirmostafaee@yahoo.com \\ 2 Department of International Environmental and Agricultural Sciences, Tokyo University of Agriculture and \\ Technology, Fuchu, Tokyo 183-8509, Japan \\ * Correspondence: azizi@um.ac.ir (M.A.); yfujii@cc.tuat.ac.jp (Y.F.); Tel.: +98-9155222478 (M.A.)
}

Received: 25 November 2019; Accepted: 14 January 2020; Published: 23 January 2020

\begin{abstract}
Medicinal and aromatic plants have the ability to transmit volatile allelochemicals and affect their surrounding organisms. In this regard, their interaction should also be considered. The inhibitory effects of 112 essential oils on lettuce seed and seedling were investigated by cotton swab method. Germination (G\%), Mean germination time (MGT), Lethal of embryo (L\%), dormancy $(\mathrm{D} \%)$, radicle growth $(\mathrm{R} \%)$, and hypocotyl growth $(\mathrm{H} \%)$ were measured. Two methods were used for evaluating allelopathic interaction effects: the simplified modified dilution check-board technique (SMCT) and the isobologram. Thymus daenensis had the highest inhibitory effect on G\% $\left(\mathrm{IC}_{50}=2.9 \mathrm{ppm}\right)$ and the most lethal effect on the embryo $\left(\mathrm{LC}_{50}=7.2 \mathrm{ppm}\right)$. Thymus transcaspicus, Dracocephalum moldavica, Artemisia sieberi and Amomum subulatum had the greatest effect on MGT. Ziziphora tenuior, Trachyspermum ammi and Pelargonium graveolens had the highest effect on $\mathrm{D} \%$. Origanum vulgare was the strongest growth inhibitor. The highest synergistic effect on $\mathrm{G} \%$ was in A. subulatum + Mentha suaveolens, on $\mathrm{H} \%$ was related to Perovskia abrotanoides $+T$. daenensis, and on $\mathrm{R} \%$ was observed in Artemisia vulgaris + M. suaveolens. The results of this study can lead to identification of new phytotoxic compounds in EOs and control weeds more effectively.
\end{abstract}

Keywords: essential oil; volatile; headspace; phytotoxic; medicinal plant; cotton swab; synergist; antagonist; isobologram; dormancy

\section{Introduction}

Growth interactions between weeds and crops lead to high costs for agricultural systems [1]. Herbicides have always played a key role in weed management. This event is one of the major causes of environmental damage and public health, leading to environmental pollution, product insecurity and human health hazards [2,3]. Furthermore, every year, a new list of pesticide-resistant weeds is released. According to the 2017 report by the International Herbicide-Resistant Weed site, a list of 36 new cases of weed resistance to herbicides was released [4]. This causes us to always look for new herbicides to control resistant weed populations.

Today, the global effort in modern agriculture is to reduce the use of harmful pesticides by introducing new biological and ecological methods. One of these methods is the use of chemical interactions among plants [5]. Interactions between plants in a common ecosystem are side effects that each plant exerts on its neighboring plants, and these include competition and allelopathy. Competition involves the active absorption of limited resources by one organism, which leads to a decrease in supply and thus to the growth inhibition of other organisms, but when one species stops growing due 
to chemicals released from another species, this mechanism is called allelopathy [6,7]. However, some researchers also consider the stimulatory effects of growth in defining allelopathy [8]. Researchers have shown that the use of allelopathic properties of some plant species has been very effective in controlling the growth of other plants, including some weeds and lettuce, which is used as an indicator plant in allelopathic bioassays [9-11]. A large number of allelochemicals from various plant species have been reported, the most important of which are phenolic compounds, benzoxazinoids, sorogoleones, glucosinolates, terpenes, alkaloids, and mamilactones [5]. Since natural herbal compounds, which are mostly safe to environment and human health, cause allelopathy, the use of allelopathy for effective weed control in agricultural systems can play an important role in environmental and community safety.

Aromatic and medicinal plants have a special place among allelopathic plants due to their secondary constituents and their active ingredients. Allelochemicals of higher plants can be released into the environment in various ways, such as volatility (predominant in dry and semi-arid conditions), leaf or stem leaching (through rain, dew, or irrigation), root secretion, and tissue degradation by microorganisms. Allelochemicals then reach the target plant by releasing to the soil, leaching, or diffusion into the air. [12]. The volatile compounds are important secondary metabolites found in medicinal and aromatic plants. Therefore, these plants are capable of transmitting allelochemical constituents in their essential oils (EOs) through diffusion into the air and thus affecting their surrounding organisms. Many studies have shown that EOs play an important role in controlling the growth of microorganisms and can effectively inhibit the growth and germination of spores of bacteria and fungi $[13,14]$. Studies have also shown that some EOs or their components effectively reduce plant growth [15]. Since EOs and volatile compounds have the potential to be used as fumigant, there will be no residue on the product if used. Therefore, these allelopathic compounds can be very effective in weed control before and after planting crops.

In the evaluation of allelopathic species, their synergistic potential can also be considered. Since plants EOs compounds may have similar or different activity, combining them may cause additive, synergistic, or antagonistic effects. Researchers have shown that there are additive and synergistic interactions between the EOs in a variety of cases, including the effects on the growth of various microorganisms as well as antioxidant effects [16-18]. So far very little research has been done on the interaction of EOs on allelopathic effects on germination and growth of other plants [19]. New research in this area could provide a new context for better understanding of allelopathic effects and achieving natural effective herbicides.

In weed science, most investigations have been done on industrial herbicides [20]. Therefore, further research is needed to identify novel methods. Finding new effective allelopathic species and their inhibitory compounds can be useful in this regard. Further studies to understand the physiological and molecular aspects of allochemicals may reveal the mechanism of allelopathic effects. This paper investigates the inhibitory effects of EOs of some medicinal and aromatic plants of Iran in gaseous phase on seed germination and seedling growth of lettuce. Also in this study, for the first time, bioassay tests were designed and implemented to investigate the allelopathic interaction.

\section{Materials and Methods}

This study was carried out in three separate experiments, and lettuce was used as test plant because it is a model plant for allelopathic bioassays owing to its short germination period and high sensitivity to phytochemicals.

\subsection{Plant Material}

Plant samples included 112 specimens of different plant organs including root, rhizome, corm, stem, leaf, flower, fruit, fruit peel, aerial part, or plant exudates such as oleogum belonging to 97 aromatic species from 16 different plant families were collected from different locations of Iran (botanical gardens, plant science research centers and natural habitats) (Table 1). In order to preserve the volatile compounds, the plants were dried according to the type of tissue (in oven at $30-60{ }^{\circ} \mathrm{C}$ 
for 1 to 3 days). EO extraction was done by hydro distillation method for $4 \mathrm{~h}$ using Clevenger-type apparatus at a distillation rate of $3 \mathrm{~mL} / \mathrm{min}$ according to the European Pharmacopoeia method [21]. The EOs were collected in dark sealed air-tight glass vials, dried with anhydrous sodium sulfate and stored in the refrigerator at $4{ }^{\circ} \mathrm{C}$.

\subsection{Evaluation of Allelopathic Effect of EOs on Lettuce Seed and Germination Characteristics}

\subsubsection{Cotton Swab Method}

To evaluate the phytotoxicity of volatile constituents on germination, 112 EOs were evaluated in two different amounts of 1 and $3 \mu \mathrm{L}$ in vial compared to the control. The experiment was conducted as a factorial experiment in completely randomized design with four replications. Allelopathic effects of volatile compounds were evaluated by cotton swab method [22] (Figure 1). For this purpose, $20 \mathrm{~mL}$ glass vials were disinfected in the oven after washing. The $0.75 \%(\mathrm{w} / \mathrm{v})$ agar solution was prepared using agar powder (Merck Inc., Kenilworth, NJ, USA) and twice distilled water and then sterilized in autoclave. Due to the high sensitivity of agar volume precision in the vial, the vial filling step was carried out after sterilization and when the agar temperature reached about $40{ }^{\circ} \mathrm{C}$. Using a graduated pipette, $10 \mathrm{~mL}$ of agar (equal to half the volume of vial) was poured into each vial. After the agar is cooled and solidified, seven lettuce seeds (Lactuca sativa, cultivar Great Lakes No. 366) were placed on the agar so that one third of the seed tip was immersed in the agar. Then a double-tipped cotton swab was cut in half and placed in the center of the agar so that its cotton tip was in the middle of the void above the agar surface. The EO was injected into a cotton swab in a specified amount (1 or $3 \mu \mathrm{L}$ ) using a Hamilton capillary syringe. Immediately, the rubber cap and aluminum seal were placed on the vial lid and sealed with Crimper (Figure 1). The vials were placed in a germinator at $21 \pm 2{ }^{\circ} \mathrm{C}$ under dark conditions. The control vials were filled with agar, seeds were sown in, the swabs were laid, then were sealed and incubated just like other vials. The only difference with the treated vials was that the swabs were not injected with essential oils.

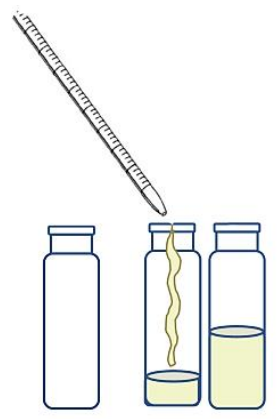

(a)

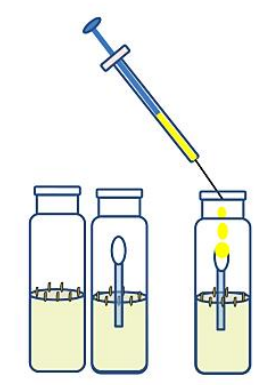

(c)

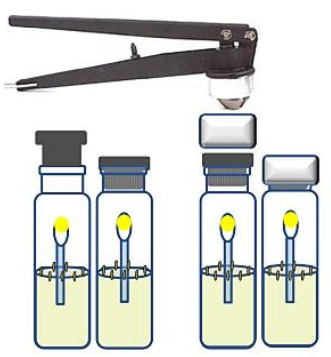

(f)

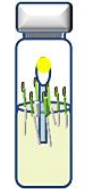

(g)

Figure 1. Schematic design of experiment to investigate the allelopathic effect of essential oils (Eos) by cotton swab method: (a) $20 \mathrm{~mL}$ vial; (b) Add $10 \mathrm{~mL}$ agar; (c) Insert lettuce seeds and cotton swabs after agar cooling and solidification; (d) Add EOs to cotton swabs using Hamilton syringe; (e) Rubber cap insertion; (f) Aluminum sealing and crimping; (g) Germination and seed growth after incubation.

Each experiment unit consisted of a vial containing seven seeds in which germination status was monitored daily for five days and germination-related traits were measured.

\subsubsection{Germination and Seed Traits}

Germination percentage (G\%) [23]:

$$
\mathrm{G} \%=\left(\frac{\mathrm{N}}{\mathrm{S}}\right) \times 100
$$

G\%: germination percentage; $N$ : total germinated seeds by the end of experiment; $S$ : total seeds. 
Mean germination time (MGT) [23]:

$$
\mathrm{MGT}=\frac{\sum \mathrm{T}_{\mathrm{i}} \mathrm{N}_{\mathrm{i}}}{\mathrm{N}}
$$

MGT: Mean germination time; $\mathrm{N}_{\mathrm{i}}$ : number of germinated seeds on day $\mathrm{T}_{\mathrm{i}} ; \mathrm{T}_{\mathrm{i}}$ : day during germination period; N: Total germinated seeds by end of experiment.

These traits were reported relative to control:

$$
\mathrm{V} \%=\frac{\mathrm{V}_{\mathrm{T}}}{\mathrm{V}_{\mathrm{C}}} \times 100
$$

$\mathrm{V} \%$ : relative value of trait; $\mathrm{V}_{\mathrm{T}}$ : average trait of treatment; $\mathrm{V}_{\mathrm{C}}$ : average trait of control.

Lethal percentage of seed embryo $(\mathrm{L} \%)$ and seed dormancy induction $(\mathrm{D} \%)$; for this purpose, the seeds that did not germinate after five days were cultured in vial without $\mathrm{EO}$ for another three days. Seeds that did not germinate after this time were uncoated and immersed in $1 \%$ tetrazolium solution $(\mathrm{pH}=7)$ and incubated for $6 \mathrm{~h}$ at $30^{\circ} \mathrm{C}$. The endosperm and embryo status were examined using a microscope and the lethality was determined (Formula no. 4). Only seeds that were completely and uniformly colored were considered as live seeds according to the ISTA instructions [24] (Figure 2). With respect to the number of germinated seeds and $L \%$, some seeds were neither germinated nor showed sign of embryo death indicating that they were dormant-induced seeds (Formula no. 5):

$$
\mathrm{L} \%=\frac{\mathrm{n}}{\mathrm{S}} \times 100
$$

L\%: Lethal percentage; n: non-colored seeds; S: total seeds.

$$
\mathrm{D} \%=\frac{\mathrm{S}-(\mathrm{N}+\mathrm{A}+\mathrm{L})}{\mathrm{S}} \times 100
$$

$\mathrm{D} \%$ : dormancy percentage; S: total seeds; N: total germinated seeds by the end of experiment; A: germinated seeds after removal of EO treatment; L: Seeds with dead embryos.

(b)

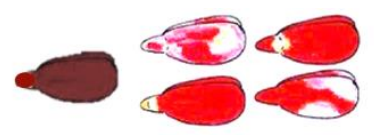

(c)

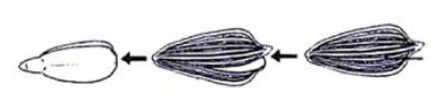

(a)

Figure 2. ISTA based embryo death assessment: (a) Seed preparation and coat removal method; (b) Unacceptable staining types that are considered abnormal seeds; (c) Uniform staining of live seed.

\subsection{Evaluation of Allelopathic Effect of EOs on Lettuce Seedling Growth}

The experiment was conducted as same as the previous one except that in this experiment germinated seeds were used to investigate the effect of EOs on lettuce seedling growth. Lettuce seeds were germinated $24 \mathrm{~h}$ prior to the experiment and those with $2 \mathrm{~mm}$ radicle were used. Each test unit was a vial containing five germinated seeds that the EO treatment was applied on and sealed. The vials were incubated at $21{ }^{\circ} \mathrm{C}$ under dark conditions for three days.

After three days, seedlings were photographed and radicle and hypocotyl length were measured using Image J software using formula no 6 and 7 respectively.

$$
\mathrm{R} \%=\frac{\mathrm{R}_{\mathrm{T}}}{\mathrm{R}_{\mathrm{C}}} \times 100
$$

$\mathrm{R} \%$ : radicle length percentage; $\mathrm{R}_{\mathrm{T}}$ : treatment radicle length; $\mathrm{R}_{\mathrm{C}}$ : control radicle length. 


$$
\mathrm{H} \%=\frac{\mathrm{H}_{\mathrm{T}}}{\mathrm{H}_{\mathrm{C}}} \times 100
$$

$\mathrm{H} \%$ : hypocotyl length percentage; $\mathrm{H}_{\mathrm{T}}$ : treatment hypocotyl length; $\mathrm{H}_{\mathrm{C}}$ : control hypocotyl length.

Then, according to germination percentage and seedling length, seedling vigor index (VI) was calculated:

$$
\mathrm{VI} \%=\mathrm{G} \% \times \mathrm{S}
$$

VI: Seedling vigor index; G\%: Germination percentage; S: Seedling length (radicle + hypocotyl).

This trait was also reported relative to control.

\subsection{Statistical Analysis}

The effect of treatments on different traits was compared and classified according to mean and standard deviation (sd) of each trait. The inhibitory effect of treatment on germination and growth increased some traits (MGT\%, D\%, $\mathrm{L} \%$ ) and decreased some others ( $\mathrm{G} \%, \mathrm{H} \%, \mathrm{R} \%$, VI\%). Intensity of inhibitory effect was considered based on the increase and decrease of these traits compare to the mean, respectively. The severity of inhibition was defined at five levels: from the highest to the least effect respectively, level five $\left(^{(* * * *)}\right.$ Mean $\pm 2 \mathrm{sd}$, level four $\left(^{* * * *}\right)$ Mean $\pm 1.5 \mathrm{sd}$, level three $\left(^{* * *}\right)$ Mean $\pm 1 \mathrm{sd}$, Level two $\left.{ }^{(* *}\right)$ Mean $\pm 0.5 \mathrm{sd}$, and level one $\left(^{*}\right)$ Mean $\pm 0.25 \mathrm{sd}$, respectively. It should be noted that MGT was not defined in some test units due to zero germination percent; for this reason, only EOs with at least three replications of germination above zero percent were compared in this trait. SPSS, Graphpad Prism and Excel were used for statistical analysis and graphing.

\subsection{Headspace Gas Chromatography-Mass Spectrometry (HS-GC-MS)}

Headspace GC-MS was performed to analyze the chemical composition of the strongest samples from the screening stage (experiment 1 and 2). For chemical analysis, 1 microlitre (equivalent to $1 \mathrm{mg}$ ) of each EO was added to the tip of a cotton swab, placed in a $20 \mathrm{~mL}$ vial then sealed and incubated at $21{ }^{\circ} \mathrm{C}$ for $1 \mathrm{~h}$. After $1000 \mu \mathrm{L}$ of head space from each vial was injected into GC-MS (Shimadzu QP 2010, Tokyo, Japan) using a $5 \mathrm{~mL}$ SGE 5MDR-HSV syringe. The injection condition was as follow: equity five capillary columns $(30 \mathrm{~m} \times 250 \mu \mathrm{m} \times 0.25 \mu \mathrm{m})$ and use of helium as carrier gas. GC-MS Operating Conditions: The temperature of the GC cooker was adjusted from 50 to $220^{\circ} \mathrm{C}$ with a rise of $3{ }^{\circ} \mathrm{C} / \mathrm{min}$, held for $10 \mathrm{~min}$ and then increased to $250{ }^{\circ} \mathrm{C}$ with a rise of $10^{\circ} \mathrm{C} / \mathrm{min}$. The compounds were identified from the mass spectra of NIST/NBS. Mass spectra were recorded at $70 \mathrm{eV}$ with a mass range of 50 to $400 \mathrm{~m} / \mathrm{z}$, compared to an internal spectral library (NIST and Wiley). Then they are validated by comparing retention times with authentic standards.

\subsection{Allelopathic Interaction Effects of EOs}

After screening the EOs the strongest inhibitors on $\mathrm{G} \%, \mathrm{~L} \%, \mathrm{R} \%, \mathrm{H} \%$ and $\mathrm{S} \%$, which caused the highest level of inhibition at $1 \mu \mathrm{L}$, were identified. In order to investigate the allelopathic interaction of these EOs, effective inhibitory and lethal concentration were first determined.

\subsubsection{Determination of $\mathrm{IC}_{50}$ and $\mathrm{LC}_{50}$}

$\mathrm{IC}_{50}$ or inhibitory concentration (the concentration of the EO that results in $50 \%$ inhibition) and $\mathrm{LC}_{50}$ or effective lethal concentration (the concentration of $\mathrm{EO}$ that causes the death of embryo in $50 \%$ of the seeds) were determined through applying different amounts of each EO (0.01 $\mu \mathrm{L}$ to $1 \mu \mathrm{L}$ per vial) so that at least five effective concentrations were in the range of $20 \%$ to $80 \%$ inhibitory. To increase the accuracy it was necessary to dilute the EOs, which was done with dimethyl sulfoxide (DMSO). The results were presented in ppm and only the void space of each vial was considered to determine the concentration. The results were analyzed using GraphPad Prism 8 software to determine $\mathrm{IC}_{50}$ and $\mathrm{LC}_{50}$. In addition, the values of $\mathrm{IC}_{25}$ and $\mathrm{IC}_{90}$, and $\mathrm{LC}_{25}$ and $\mathrm{LC}_{90}$ were calculated with Quick Calcs online software. The most effective EOs were identified after determination of $\mathrm{IC}_{50}$ and $\mathrm{LC}_{50}$. 


\subsubsection{Essential oils Combinations}

Some of the most effective EOs were selected and their combination effects were evaluated. The experiment was conducted as factorial in a completely randomized design with four replications. Simplified modified dilution check-board technique (SMCT) in cotton swab method was used and two different EOs (A and B) were evaluated at their $\mathrm{IC}_{25}$ concentration. Experiments were performed separately on seed germination, radicle growth, and hypocotyl growth. For germination evaluation the method was similar to the first experiment, and for the radicle and hypocotyl growth it was done like second experiment. The allelopathic interaction effect of the EOs was determined using two methods: determination of the combination effect (CE), and drawing the isobologram curves.

- Combination effect (CE) was calculated using the following equation:

$$
C E_{(A, B)}=\frac{I_{\left(A_{25}+B_{25}\right)}}{\left[\mathrm{I}_{\left(2 A_{25}\right)}+I_{\left(2 B_{25}\right)}\right] / 2}
$$

$\mathrm{CE}_{(\mathrm{A}, \mathrm{B})}$ : combination effect of two EOs (A and B); I: Inhibitory percentage; $\mathrm{A}_{25}$ and $\mathrm{B}_{25}$ : $\mathrm{A}$ and $\mathrm{B}$ in their $\mathrm{IC}_{25}$ concentration; $2 \mathrm{~A}_{25}$ and $2 \mathrm{~B}_{25}$ : $\mathrm{A}$ and $\mathrm{B}$ in their twice $\mathrm{IC}_{25}$ concentration (calculated using Graphpad prism).

In this equation if $\mathrm{CE} \geq 1.1$ then the interaction was considered as synergistic effect, if $0.9<\mathrm{CE}$ $<1.1$ was then considered as additive effect, and if $\mathrm{CE} \leq 0.9$ then the interaction was considered as antagonistic effect.

- Isobologram curves

To draw these curves, the inhibitory effect of two EOs (A and B) was considered as the basis, then the concentration of $A$ and $B$ independently leading to similar inhibition was calculated using Quick Calcs software. Thus, there were three concentrations (A alone, B alone, and A or B in combination state) for a fixed amount of inhibition (inhibition caused by the combination of two EOs), using in isobologram curve. This curve can have three different states: (1) no curvature means additive effect, (2) upward curvature means antagonistic effect, and (3) downward curvature means synergistic effect (Figure 3).

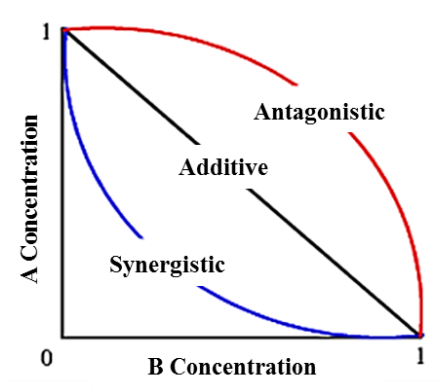

Figure 3. Schematic isobologram curve for determination of allelopathic interactions.

\section{Results}

\subsection{Allelopathic Effects of Essential Oils on Lettuce Seed and Germination Characteristics}

All germination traits were significantly affected by plant EO treatment at both amount of 1 and $3 \mu \mathrm{L}$ (Table 1). 
Table 1. The results of evaluation of inhibitory potential of medicinal plants EO on traits related to seed germination and seedling growth of lettuce.

\begin{tabular}{|c|c|c|c|c|c|c|c|c|c|c|c|c|c|c|c|c|c|}
\hline \multirow{2}{*}{ No. } & \multirow{2}{*}{ Plant Scientific Name } & \multirow{2}{*}{ Plant Family } & \multirow{2}{*}{$\begin{array}{l}\text { Part of } \\
\text { Use }^{\mathrm{a}}\end{array}$} & \multicolumn{2}{|c|}{$\mathrm{G}^{\mathrm{b}}{ }^{\mathrm{b}}$} & \multicolumn{2}{|c|}{ MGT (Day) ${ }^{b}$} & \multicolumn{2}{|c|}{$\mathrm{D}^{\mathrm{b}} \mathrm{b}$} & \multicolumn{2}{|c|}{$\mathrm{L}^{\mathrm{b}} \mathrm{b}^{\mathrm{b}}$} & \multicolumn{2}{|c|}{$\mathrm{H}^{\mathrm{b}}$} & \multicolumn{2}{|c|}{$R^{2}{ }^{b}$} & \multicolumn{2}{|c|}{$\mathrm{VI}^{\mathrm{b}} \mathrm{b}$} \\
\hline & & & & $1 \mu \mathrm{L}$ & $3 \mu \mathrm{L}$ & $1 \mu \mathrm{L}$ & $3 \mu \mathrm{L}$ & $1 \mu \mathrm{L}$ & $3 \mu \mathrm{L}$ & $1 \mu \mathrm{L}$ & $3 \mu \mathrm{L}$ & $1 \mu \mathrm{L}$ & $3 \mu \mathrm{L}$ & $1 \mu \mathrm{L}$ & $3 \mu \mathrm{L}$ & $1 \mu \mathrm{L}$ & $3 \mu \mathrm{L}$ \\
\hline 1 & Abies alba & Pinaceae & $\mathrm{L}$ & 100 & 100 & 3.3 & 2.5 & 0 & 0 & 0 & 0 & 27.8 & 43.2 & 42.7 & 63.9 & 36.9 & 56 \\
\hline 2 & Achillea filipendulina & Asteraceae & Ap & 60.7 & 96.5 & $4.3^{* * *}$ & 2.5 & 0 & 0 & 3.6 & 0 & $10.8^{* *}$ & $25.8^{* *}$ & $5.7 * *$ & $16.6^{* * *}$ & $4.7 * *$ & $19.4 * *$ \\
\hline 3 & Achillea wilhelmsii & Asteraceae & $A p$ & 60.7 & 93 & $4.8^{* * * *}$ & $3.7 * *$ & 0 & 0 & 3.6 & 0 & $12.1^{* *}$ & $21.2^{* *}$ & $4.8^{* *}$ & $18.2 * *$ & 4.6 ** & $18.0^{* *}$ \\
\hline 4 & Allium hirtifolium & Liliaceae & C & $0.0^{* * * *}$ & $3.5^{* * * * *}$ & & 5 & 0 & 0 & $100^{* * * * *}$ & $96.4^{* * * * *}$ & $6.9^{* *}$ & $10.4^{* * *}$ & $1.2 * *$ & $5.2^{* * *}$ & $0.0^{* *}$ & $0.3^{* * *}$ \\
\hline 5 & Amomum subulatum & Zingiberaceae & $\mathrm{Fr}$ & $0.0^{* * * *}$ & $28.5^{* * * *}$ & & $4.8^{* * * * *}$ & 3.6 & 0 & $96.4^{* * * * *}$ & 0 & $0.8^{* * *}$ & $12.2^{* * *}$ & $1.0^{* *}$ & $10.8^{* * *}$ & $0.0^{* *}$ & $3.2 * * *$ \\
\hline 6 & Anethum graveolens & Apiaceae & $\mathrm{Fr}$ & $21.4^{* * *}$ & 92.8 & $4.7^{* * * *}$ & $3.8^{* *}$ & $57.1^{* * * * *}$ & 0 & 0 & 0 & $4.4^{* * *}$ & $14.6^{* * *}$ & $0.5^{* *}$ & $18.0^{* *}$ & $0.4^{* *}$ & $15.5^{* *}$ \\
\hline 7 & Apium graveolens & Apiaceae & $\mathrm{Fr}$ & 100 & 89.3 & 2.2 & 2.3 & 0 & 0 & 0 & 3.6 & 22.2 & $29.2 *$ & 59.9 & 61.2 & 45.4 & 43.7 \\
\hline 8 & Artemisia absinthium & Asteraceae & $\mathrm{L}$ & 96.4 & 100 & 3.2 & 2.5 & 0 & 0 & 0 & 0 & 28.7 & 42.2 & 44.9 & 49.9 & 37.3 & 46.9 \\
\hline 9 & Artemisia aucheri & Asteraceae & Ap & 82.1 & 100 & $4.7^{* * * * *}$ & $3.8^{* *}$ & 0 & 0 & 0 & 0 & $6.9 * *$ & $23.0^{* *}$ & $0.0 * * *$ & $13.8^{* * *}$ & $2.2^{* *}$ & $17.3^{* *}$ \\
\hline 10 & Artemisia deserti & Asteraceae & $\mathrm{Ap}$ & 89.3 & 100 & $3.9^{* *}$ & $3.3 *$ & 0 & 0 & 0 & 0 & $7.1^{* *}$ & $21.0^{* *}$ & $0.0 * * *$ & $16.7^{* *}$ & $2.4^{* *}$ & $18.4^{* *}$ \\
\hline 11 & Artemisia dracunculus & Asteraceae & $\mathrm{L}$ & 100 & 100 & 2.5 & 2.1 & 0 & 0 & 0 & 0 & 20.8 & 39.9 & $17.5 *$ & 43.6 & 18.8 & 42.2 \\
\hline 12 & Artemisia ludoviciana & Asteraceae & $\mathrm{L}$ & $10.7^{* * *}$ & 96.5 & 4.8 & $3.9^{* * *}$ & 7.2 & 0 & 7.2 & 0 & $4.7^{* * *}$ & $14.1^{* * *}$ & $1.3^{* *}$ & $3.7^{* * * *}$ & $0.3^{* *}$ & $7.4^{* * *}$ \\
\hline 13 & Artemisia scoparia & Asteraceae & $\mathrm{L}$ & $25.0^{* *}$ & 82.3 & $4.8^{* * * *}$ & $4.5^{* * * *}$ & 3.6 & 3.6 & 0 & 7.2 & 10.8 & $19.9^{* *}$ & $2.3^{* *}$ & $16.9^{* *}$ & $1.4^{* *}$ & $14.9^{* *}$ \\
\hline 14 & Artemisia turanies & Asteraceae & Ap & 82.1 & 100 & $4.3^{* * *}$ & $3.6^{* *}$ & 0 & 0 & 3.6 & 0 & 19.1 * & 35.8 & 20.2 & 48.6 & 16.2 & 43.7 \\
\hline 15 & Artemisia vulgaris & Asteraceae & $\mathrm{L}$ & 85.7 & 100 & 3.4 & 2.6 & 0 & 0 & 3.6 & 0 & 24.7 & 45.1 & $14.8^{*}$ & $33.8^{*}$ & 15.9 & 38.1 \\
\hline 16 & Artemisia vulgaris & Asteraceae & $\mathrm{F}$ & 89.5 & 100 & $4.5^{* * * *}$ & $3.9^{* * *}$ & 0 & 0 & 7.2 & 0 & $12.2^{* *}$ & $18.4^{* *}$ & $5.4^{* *}$ & $7.3^{* * *}$ & $7.2 * *$ & $11.6^{* * *}$ \\
\hline 17 & Artemisia sieberi & Asteraceae & $\mathrm{L}, \mathrm{S}$ & $0.0^{* * * *}$ & $50.0^{* * *}$ & & $4.7^{* * * *}$ & 7.2 & 0 & 17.9 & 7.2 & $11.3^{* *}$ & $20.7^{* *}$ & $0.6^{* *}$ & $7.6^{* * *}$ & 0.0 ** & $6.3^{* * *}$ \\
\hline 18 & Ballota nigra & Lamiaceae & $\mathrm{L}, \mathrm{F}$ & 100 & 92.8 & 2.8 & 3 & 0 & 0 & 0 & 0 & 83.2 & 93.5 & 78.9 & 90.1 & 80.6 & 84.9 \\
\hline 19 & Bunium persicum & Apiaceae & $\mathrm{Fr}$ & $0.0^{* * * *}$ & $25.3^{* * * *}$ & & $4.5^{* * * *}$ & 0 & 0 & $89.3^{* * * * *}$ & 10.7 & $11.2^{* *}$ & $30.6 *$ & $4.3^{* *}$ & $33.1 *$ & $0.0^{* *}$ & $8.0 * * *$ \\
\hline 20 & Cedrus atlantica & Pinaceae & $\mathrm{L}$ & 96.4 & 96.5 & 3.1 & 2.7 & 3.6 & 0 & 0 & 0 & 40.8 & 44.3 & 63.9 & 67.6 & 53.1 & 56.5 \\
\hline 21 & Chamaecyparis lawsoniana & Cupressaceae & $\mathrm{L}$ & 57.1 & 93 & $4.8^{* * * *}$ & $3.7^{* *}$ & 0 & 0 & 3.6 & 0 & 20.9 & 42.8 & 27.4 & 37.5 & $14.2^{*}$ & 36.7 \\
\hline 22 & Chamaecyparis lawsoniana & Cupressaceae & $\mathrm{L}$ & 96.4 & 100 & 2 & 2 & 0 & 0 & 0 & 0 & 23.5 & 33.8 & 30.7 & 51.2 & 27 & 44.5 \\
\hline 23 & Chamaecyparis sp. & Cupressaceae & $\mathrm{L}$ & 96.4 & 96.5 & 3 & 2.8 & 0 & 0 & 0 & 0 & $18.6^{*}$ & 34 & $9.3 * *$ & $24.3^{* *}$ & 12.4 * & $27.0^{*}$ \\
\hline 24 & Chenopodium botrys & Chenopodiaceae & $\mathrm{L}$ & 100 & 93 & 3.1 & 3.1 & 0 & 0 & 0 & 3.6 & 46 & 50 & 66.1 & 66.7 & 58.4 & 56 \\
\hline 25 & Chrysanthemum morifolium & Asteraceae & $\mathrm{L}$ & 92.9 & 96.5 & 3.8 ** & $3.3^{*}$ & 3.6 & 0 & 0 & 0 & $18.4^{*}$ & 35.3 & $4.2^{* *}$ & $33.3 *$ & 9.0 ** & 32.8 \\
\hline 26 & Citrus $\times$ limon & Rutaceae & $\mathrm{Fp}$ & 92.9 & 96.5 & $3.7^{*}$ & 2.4 & 0 & 3.6 & 0 & 0 & 40.2 & 67.6 & 59.7 & 90.5 & 48.4 & 78.8 \\
\hline 27 & Citrus $\times$ paradisi & Rutaceae & $\mathrm{Fp}$ & 92.9 & 100 & 2.4 & 2.3 & 3.6 & 0 & 0 & 0 & 26.7 & 46.1 & 24.5 & 57.8 & 23.5 & 53.3 \\
\hline 28 & Citrus $\times$ sinensis & Rutaceae & $\mathrm{Fp}$ & 100 & 96.5 & 2.7 & 2.3 & 0 & 0 & 0 & 0 & 29.4 & 71.1 & 37.4 & 85.9 & 34.3 & 77.3 \\
\hline 29 & Citrus aurantifolia & Rutaceae & $\mathrm{Fp}$ & 100 & 100 & 2.5 & 2.2 & 0 & 0 & 0 & 0 & 28.8 & 64.3 & $15.6^{*}$ & 53.4 & 20.7 & 57.6 \\
\hline 30 & Citrus aurantifolia & Rutaceae & $\mathrm{L}$ & $0.0^{* * * *}$ & $29.0^{* * * *}$ & & $4.5^{* * * *}$ & $25.0^{* * * *}$ & 3.6 & $39.3 * *$ & 3.6 & 23.1 & $30.6 *$ & $13.3^{*}$ & 45.2 & 0.0 ** & $11.3^{* * *}$ \\
\hline 31 & Citrus aurantium & Rutaceae & $\mathrm{Fp}$ & 100 & 100 & 2.2 & 2 & 0 & 0 & 0 & 0 & 54.6 & 70.9 & 72.6 & 74.3 & 65.7 & 73 \\
\hline 32 & Citrus japonica & Rutaceae & $\mathrm{Fp}$ & 92.9 & 96.5 & 2.2 & 2.3 & 3.6 & 3.6 & 0 & 0 & 32.8 & 56.2 & 54.1 & 87 & 42.6 & 72.5 \\
\hline 33 & Citrus medica & Rutaceae & $\mathrm{Fp}$ & 92.9 & 96.5 & 3 & 2.6 & 7.2 & 3.6 & 0 & 0 & 45.2 & 58.5 & 77.5 & 76.8 & 60.4 & 67.2 \\
\hline 34 & Citrus reticulata & Rutaceae & $\mathrm{Fp}$ & 100 & 93 & 2.4 & 2.3 & 0 & $7.2^{*}$ & 0 & 0 & 35.3 & 60.6 & 35.5 & 61.2 & 35.4 & 56.6 \\
\hline 35 & Citrus $\times$ tangelo & Rutaceae & $\mathrm{Fp}$ & 100 & 100 & 2.1 & 2.1 & 0 & 0 & 0 & 0 & 48.6 & 71.4 & 57.5 & 85 & 54.1 & 79.7 \\
\hline 36 & Citrus $\times$ latifolia & Rutaceae & $\mathrm{Fp}$ & 89.3 & 100 & 3.3 & 2.8 & 0 & 0 & 0 & 0 & $15.6^{*}$ & $28.2 *$ & $14.3^{*}$ & $34.1 *$ & $13.2 *$ & 31.8 \\
\hline 37 & Coffea arabica & Rubiaceae & $\mathrm{Fr}$ & 89.3 & 100 & 2.5 & 2.1 & 0 & 0 & 10.7 & 0 & 85.4 & 94.9 & 94.2 & 106.9 & 81.1 & 102.3 \\
\hline 38 & Coriandrum sativum & Apiaceae & $\mathrm{Fr}$ & $50.0 *$ & 93 & $4.2 * *$ & 3.1 & 0 & 0 & 0 & 0 & $6.0 * *$ & $18.8^{* *}$ & $1.9^{* *}$ & $24.9^{* *}$ & $1.7 * *$ & $20.9^{* *}$ \\
\hline 39 & Ситіпит сутіпит & Apiaceae & $\mathrm{Fr}$ & $3.6^{* * * *}$ & 86 & 5 & $3.8^{* *}$ & 3.6 & 0 & 14.3 & 3.6 & $11.6^{* *}$ & $23.2^{* *}$ & $6.0 *$ & $21.2^{* *}$ & 0.3 ** & $18.9^{* *}$ \\
\hline 40 & Cupressus arizonica & Cupressaceae & $\mathrm{L}$ & 89.3 & 96.5 & $4.3^{* * *}$ & $3.7^{* *}$ & 0 & 3.6 & 3.6 & 0 & 21.8 & 39.6 & $19.7 *$ & 47.5 & 18.3 & 42.8 \\
\hline 41 & Cupressus arizonica & Cupressaceae & $\mathrm{Fr}$ & 67.9 & 100 & 3.4 & 3.1 & 0 & 0 & 0 & 0 & 37.5 & 47 & 74.7 & 86.1 & 41 & 71.1 \\
\hline 42 & Cupressus sempervirens & Cupressaceae & $\mathrm{L}$ & 100 & 89.3 & $3.6^{*}$ & 2.7 & 0 & 0 & 0 & 7.2 & 38.2 & 51.1 & 73.2 & 91.8 & 59.7 & 68 \\
\hline
\end{tabular}


Table 1. Cont.

\begin{tabular}{|c|c|c|c|c|c|c|c|c|c|c|c|c|c|c|c|c|c|}
\hline \multirow{2}{*}{ No. } & \multirow{2}{*}{ Plant Scientific Name } & \multirow{2}{*}{ Plant Family } & \multirow{2}{*}{$\begin{array}{l}\text { Part of } \\
\text { Use }^{\mathbf{a}}\end{array}$} & \multicolumn{2}{|c|}{$\mathrm{G}^{\mathrm{b}}{ }^{\mathrm{b}}$} & \multicolumn{2}{|c|}{ MGT (Day) ${ }^{b}$} & \multicolumn{2}{|c|}{$\mathrm{D}^{\mathrm{b}}{ }^{\mathrm{b}}$} & \multicolumn{2}{|c|}{$\mathrm{L}^{\mathrm{b}}$} & \multicolumn{2}{|c|}{$\mathrm{H}^{\mathrm{b}}{ }^{\mathrm{b}}$} & \multicolumn{2}{|c|}{$\mathbf{R}^{\mathrm{b}}$} & \multicolumn{2}{|c|}{$\mathrm{VI} \% \mathrm{~b}$} \\
\hline & & & & $1 \mu \mathrm{L}$ & $3 \mu \mathrm{L}$ & $1 \mu \mathrm{L}$ & $3 \mu \mathrm{L}$ & $1 \mu \mathrm{L}$ & $3 \mu \mathrm{L}$ & $1 \mu \mathrm{L}$ & $3 \mu \mathrm{L}$ & $1 \mu \mathrm{L}$ & $3 \mu \mathrm{L}$ & $1 \mu \mathrm{L}$ & $3 \mu \mathrm{L}$ & $1 \mu \mathrm{L}$ & $3 \mu \mathrm{L}$ \\
\hline 43 & Curcuma longa & Zingiberaceae & $\mathrm{Rh}$ & 60.7 & 86 & $3.8^{* *}$ & $3.8^{* *}$ & 3.6 & 0 & 0 & 0 & 47.1 & 49.2 & 91.8 & 99.5 & 45.3 & 68.7 \\
\hline 44 & Datura stramonium & Solanaceae & $\mathrm{L}$ & 100 & 96.5 & 3 & $3.3^{*}$ & 0 & 0 & 0 & 0 & 73.1 & 72.7 & 65.4 & 76.6 & 68.3 & 72.4 \\
\hline 45 & Daucus carota & Apiaceae & $\mathrm{Fr}$ & 92.9 & 96.5 & 2.9 & 2.6 & 0 & 0 & 3.6 & 0 & 19.6 & $27.1 *$ & $12.8^{* *}$ & $28.7^{* *}$ & $14.3 *$ & $27.1 *$ \\
\hline 46 & Dracocephalum moldavica & Lamiaceae & Ap & $0.0^{* * * *}$ & $39.5^{* * *}$ & & $4.7^{* * * * *}$ & 0 & 0 & $92.9^{* * * *}$ & 7.2 & $4.8^{* * *}$ & $24.2 * *$ & $0.7^{* *}$ & $23.8^{* *}$ & 0.0 ** & $9.4^{* * *}$ \\
\hline 47 & Durema ammonicum & Apiaceae & $\mathrm{O}$ & 92.9 & 100 & 2.4 & 2.4 & 0 & 0 & 0 & 0 & 37 & 49.6 & 41.5 & 71.8 & 36.9 & 63.3 \\
\hline 48 & Elettaria cardamomum & Zingiberaceae & $\mathrm{Fr}$ & 100 & 96.5 & 3.3 & 2.4 & 0 & 3.6 & 0 & 0 & $12.9^{* *}$ & $27.1 *$ & $11.0^{* *}$ & 38.2 & $11.8^{*}$ & 32.7 \\
\hline 49 & Eucalptus globulus & Myrtaceae & L3 & 100 & 89.3 & 3.4 & 2.6 & 0 & 0 & 0 & 7.2 & 20.6 & 32.2 & 24.2 & 46.1 & 22.8 & 36.4 \\
\hline 50 & Ferula alliacea & Apiaceae & Ap & 96.4 & 100 & 3.1 & 2.6 & 0 & 0 & 3.6 & 0 & 24 & 43 & 23.6 & 51.9 & 22.9 & 48.5 \\
\hline 51 & Ferula foetida & Apiaceae & $\mathrm{O}$ & $0.0^{* * * *}$ & 93 & & 3.2 & 0 & 3.6 & $100^{* * * * *}$ & 0 & $9.3 * *$ & 38.5 & $4.7^{* *}$ & 48.1 & $0.0^{* *}$ & 41.3 \\
\hline 52 & Ferula gumosa & Apiaceae & $\mathrm{O}$ & 100 & 100 & 2.6 & 2.6 & 0 & 0 & 0 & 0 & 42.2 & 76 & 54.8 & 88.3 & 50 & 83.6 \\
\hline 53 & Ferula lutensis & Apiaceae & $\mathrm{L}$ & 89.3 & 100 & 2.9 & 2.3 & 0 & 0 & 3.6 & 0 & $17.8^{*}$ & 32 & $10.9^{* *}$ & $30.7^{*}$ & 12.1 * & 31.2 \\
\hline 54 & Foeniculum vulgare & Apiaceae & $\mathrm{Fr}$ & 100 & 100 & 3.2 & 2.5 & 0 & 0 & 0 & 0 & $9.0 * *$ & $29.8 *$ & $1.8^{* *}$ & $32.2 *$ & $4.6^{* *}$ & 31.3 \\
\hline 55 & Foeniculum vulgare & Apiaceae & $\mathrm{L}$ & 78.6 & 100 & $3.9^{* *}$ & 3.1 & 0 & 0 & 3.6 & 0 & 25.6 & 42.2 & 29.1 & 48.4 & 21.8 & 46.1 \\
\hline 56 & Grindelia robusta & Asteraceae & $\mathrm{L}, \mathrm{F}$ & 92.9 & 93 & 2.8 & 2.4 & 3.6 & 0 & 0 & 0 & 26.6 & 51.7 & 31 & 72.4 & 27.2 & 59.8 \\
\hline 57 & Helianthus annuиs & Asteraceae & $\mathrm{L}$ & 100 & 100 & 2.8 & 2.4 & 0 & 0 & 0 & 0 & 26.9 & 47.5 & $17.4^{*}$ & 63.1 & 21.1 & 57.1 \\
\hline 58 & Helichrysum italicum & Asteraceae & Ap & $53.6^{*}$ & 96.5 & $4.1^{* *}$ & $3.6^{* *}$ & 0 & 0 & 3.6 & 0 & 19.4 & $31.1 *$ & 27.4 & 42.4 & $13.0^{*}$ & 36.7 \\
\hline 59 & Heracleum persicum & Apiaceae & $\mathrm{Fr}$ & $25.0^{* *}$ & 100 & 3.3 & 2.4 & $14.3^{* *}$ & 0 & 3.6 & 0 & $7.5^{* *}$ & $29.6^{*}$ & 3.5 & $30.1 *$ & $1.3 * *$ & $29.9 *$ \\
\hline 60 & Inula paecockianum & Asteraceae & $\mathrm{R}$ & 100 & 93 & 2 & 1.7 & 0 & 0 & 0 & 0 & 71.4 & 70.7 & 75.1 & 74 & 73.7 & 67.5 \\
\hline 61 & Juniperus chinensis & Cupressaceae & $\mathrm{L}$ & 92.9 & 96.5 & 3.3 & 2.9 & 0 & 3.6 & 0 & 0 & 19.4 & $27.7 *$ & 23.4 & 39.2 & 20.3 & 33.5 \\
\hline 62 & Juniperus excelsa & Cupressaceae & $\mathrm{L}$ & 92.9 & 100 & 2 & 2.2 & 7.2 & 0 & 0 & 0 & 42.9 & 61.5 & 73.8 & 91.9 & 57.5 & 80.2 \\
\hline 63 & Juniperus horizontalis & Cupressaceae & $\mathrm{Fr}$ & 96.5 & 100 & 2.4 & 2.6 & 0 & 0 & 0 & 0 & 45.9 & 56.5 & 59.7 & 69.5 & 52.5 & 64.5 \\
\hline 64 & Juniperus horizontalis & Cupressaceae & $\mathrm{L}$ & 96.4 & 92.8 & 2.1 & 2 & 3.6 & 3.6 & 0 & 0 & $15.6^{*}$ & $31.2 *$ & 22.7 & 53.3 & 19.2 & 41.6 \\
\hline 65 & Juniperus sp. & Cupressaceae & $\mathrm{L}, \mathrm{F}$ & 100 & 96.5 & 2.8 & 2.4 & 0 & 3.6 & 0 & 0 & 33.3 & 38.3 & 37 & $36.2 *$ & 35.6 & 35.7 \\
\hline 66 & Lantana camara & Verbenaceae & $\mathrm{L}, \mathrm{F}$ & 92.9 & 93 & 2.9 & 2.4 & 3.6 & 0 & 0 & 0 & 32.4 & 39.1 & 53.7 & 47.5 & 42.2 & 41.1 \\
\hline 67 & Lavandula angustifolia & Lamiaceae & $\mathrm{L}$ & 71.4 & 93 & $3.9^{* *}$ & $3.5^{* *}$ & 0 & 0 & 3.6 & 3.6 & $15.4^{*}$ & $27.5 *$ & $9.3^{* *}$ & 45.6 & $8.3^{* *}$ & 35.9 \\
\hline 68 & Lavandula angustifolia & Lamiaceae & $\mathrm{F}$ & $21.4^{* * *}$ & 96.5 & $4.1^{* *}$ & $3.8 * *$ & 0 & 0 & 3.6 & 0.0 & $2.0^{* * *}$ & $13.1^{* * *}$ & $0.2 * * *$ & $4.1^{* * *}$ & $0.2 * *$ & $7.3^{* * *}$ \\
\hline 69 & Lippia citriodora & Verbenaceae & $\mathrm{L}$ & 60.7 & 96.5 & $4.4^{* * *}$ & $3.3 *$ & 0 & 0 & 10.7 & 0 & $7.3^{* *}$ & 33 & $5.8^{* *}$ & $24.3^{* *}$ & $3.9^{* *}$ & $26.6 *$ \\
\hline 70 & Magnolia virginiana & Magnoliaceae & $\mathrm{L}$ & 96.4 & 85.8 & 2.7 & 3.1 & 3.6 & 0 & 0 & 10.7 & 63.6 & 62.1 & 70.2 & 70.7 & 65.2 & 57.8 \\
\hline 71 & Melissa officinalis & Lamiaceae & $\mathrm{L}$ & $0.0^{* * * *}$ & $3.5^{* * * * *}$ & & 4 & 0 & 0 & $100^{* * * * *}$ & $21.5^{* *}$ & $15.0^{*}$ & $21.1^{* *}$ & $6.3^{* *}$ & $18.8^{* *}$ & $0.0^{* *}$ & $0.7^{* * *}$ \\
\hline 72 & Mentha longifolia & Lamiaceae & $\mathrm{L}$ & $53.6^{*}$ & 96.5 & $4.3^{* * * *}$ & $3.6^{* *}$ & 0 & 0 & 0 & 0 & $11.8^{* *}$ & $19.0^{* *}$ & $5.2^{* *}$ & $14.3^{* * *}$ & $4.1^{* *}$ & $15.5^{* *}$ \\
\hline 73 & Mentha piperita & Lamiaceae & $\mathrm{L}, \mathrm{F}$ & $32.2 * *$ & $60.5^{* *}$ & $4.4^{* * *}$ & $4.2^{* * *}$ & $17.9^{* * *}$ & 3.6 & 0 & 3.6 & $9.4 * *$ & $26.8^{* *}$ & $4.7^{* *}$ & $35.6^{*}$ & $2.1 * *$ & $19.6^{* *}$ \\
\hline 74 & Mentha pulegium & Lamiaceae & $\mathrm{L}, \mathrm{F}$ & $53.6^{*}$ & 96.5 & $4.4^{* * *}$ & 3.7 & 7.2 & 0 & 0 & 0 & $7.3^{* *}$ & $21.3^{* *}$ & $3.3^{* *}$ & $20.2^{* *}$ & $2.6^{* *}$ & $19.9^{* *}$ \\
\hline 75 & Mentha suaveolens & Lamiaceae & $\mathrm{L}$ & $0.0^{* * * *}$ & 10.8 & & 4.5 & 0 & 14.3 & $92.9^{* * * * *}$ & 3.6 & $12.2^{* *}$ & $15.8^{* * *}$ & $6.5^{* *}$ & $7.6^{* * *}$ & $0.0^{* *}$ & $1.2^{* * *}$ \\
\hline 76 & Microcephala lamellata & Asteraceae & $\mathrm{L}, \mathrm{F}$ & 89.3 & 100 & 2.7 & 2.3 & 0 & 0 & 3.6 & 0 & 39.5 & 46.4 & 43.5 & 75.4 & 37.5 & 64.2 \\
\hline 77 & Myristica fragrans & Myrtaceae & $\mathrm{Fr}$ & 96.4 & 100 & 3.1 & 2.5 & 0 & 0 & 0 & 0 & 25.3 & 41.8 & 38.2 & 54.5 & 32 & 49.6 \\
\hline 78 & Nepeta binaludensis & Lamiaceae & Ap & $10.7 * * *$ & 78.5 & 5 & $4.6^{* * * *}$ & 3.6 & 3.6 & 3.6 & 0 & 26.9 & 57.4 & 38.2 & 66.6 & $3.6^{* *}$ & 49.5 \\
\hline 79 & Nepeta cataria & Lamiaceae & $\mathrm{L}, \mathrm{F}$ & $14.3^{* * *}$ & $68.0^{*}$ & 4.3 & $4.4^{* * * *}$ & $42.9^{* * * * *}$ & 3.6 & 14.3 & 0 & 24.8 & 55.4 & $10.9^{* *}$ & 54.7 & $2.3^{* *}$ & 37.3 \\
\hline 80 & $\begin{array}{c}\text { Origanum vulgare subsp. } \\
\text { viridi }\end{array}$ & Lamiaceae & $\mathrm{F}$ & $46.4^{*}$ & 89.5 & $4.5^{* * *}$ & $3.3^{*}$ & 0 & 0 & 7.2 & 3.6 & $5.6^{* * *}$ & $5.0^{* * * *}$ & $0.1^{* * *}$ & $2.3^{* * * *}$ & $1.0^{* *}$ & $3.0^{* * *}$ \\
\hline 81 & $\begin{array}{c}\text { Origanum vulgare subsp. } \\
\text { vulgare }\end{array}$ & Lamiaceae & $\mathrm{L}$ & $0.0^{* * * *}$ & $7.0^{* * * * *}$ & & 5 & 0 & 0 & $100^{* * * * *}$ & $85.7^{* * * * *}$ & $4.6^{* * *}$ & $10.5^{* * *}$ & $0.2 * * *$ & $8.4^{* * *}$ & $0.0^{* *}$ & $0.7^{* * *}$ \\
\hline
\end{tabular}


Table 1. Cont.

\begin{tabular}{|c|c|c|c|c|c|c|c|c|c|c|c|c|c|c|c|c|c|}
\hline \multirow{2}{*}{ No. } & \multirow{2}{*}{ Plant Scientific Name } & \multirow{2}{*}{ Plant Family } & \multirow{2}{*}{$\begin{array}{l}\text { Part of } \\
\text { Use }^{a}\end{array}$} & \multicolumn{2}{|c|}{$\mathrm{G}^{\mathrm{b}}$} & \multicolumn{2}{|c|}{ MGT (Day) $^{b}$} & \multicolumn{2}{|c|}{$\mathrm{D}^{\mathrm{b}}{ }^{\mathrm{b}}$} & \multicolumn{2}{|c|}{$\mathbf{L}^{\mathrm{b}}$} & \multicolumn{2}{|c|}{$\mathrm{H}^{\mathrm{b}}{ }^{\mathrm{b}}$} & \multicolumn{2}{|c|}{$\mathbf{R} \%^{b}$} & \multicolumn{2}{|c|}{$\mathrm{VI} \%^{b}$} \\
\hline & & & & $1 \mu \mathrm{L}$ & $3 \mu \mathrm{L}$ & $1 \mu \mathrm{L}$ & $3 \mu \mathrm{L}$ & $1 \mu \mathrm{L}$ & $3 \mu \mathrm{L}$ & $1 \mu \mathrm{L}$ & $3 \mu \mathrm{L}$ & $1 \mu \mathrm{L}$ & $3 \mu \mathrm{L}$ & $1 \mu \mathrm{L}$ & $3 \mu \mathrm{L}$ & $1 \mu \mathrm{L}$ & $3 \mu \mathrm{L}$ \\
\hline 82 & $\begin{array}{c}\text { Origanum vulgare subsp. } \\
\text { vulgare }\end{array}$ & Lamiaceae & $\mathrm{F}$ & 100 & 100 & 2.2 & 2.1 & 0 & 0 & 0 & 0 & 44.1 & 45.3 & 61 & 62.1 & 54.5 & 55.7 \\
\hline 83 & Pelargonium graveolnes & Geraniaceae & $\mathrm{L}$ & $3.6^{* * * *}$ & $7.0^{* * * * *}$ & 5 & 3.5 & $25.0^{* * * *}$ & $\begin{array}{l}57.1 \\
* * * * * *\end{array}$ & $71.4^{* * * *}$ & 0 & $14.3^{* *}$ & $19.0^{* *}$ & 26.6 & $27.8^{* *}$ & $0.8^{* *}$ & $1.7^{* * *}$ \\
\hline 84 & Pelargonium graveolnes & Geraniaceae & $\mathrm{L}, \mathrm{F}$ & $0.0^{* * * *}$ & $0.0^{* * * * *}$ & & & $14.3^{* *}$ & 32.2 & $82.1^{* * * * *}$ & $32.2^{* * *}$ & $16.4^{*}$ & $13.7^{* * *}$ & $18.4^{*}$ & $25.6^{* *}$ & $0.0^{* *}$ & $0.0^{* * *}$ \\
\hline 85 & Perovskia abrotanoides & Lamiaceae & $\mathrm{F}$ & $53.6^{*}$ & 89.3 & $4.8^{* * * *}$ & $3.8^{* *}$ & 3.6 & 0 & 0 & 0 & $7.8^{* *}$ & $6.7^{* * * * *}$ & $1.0^{* *}$ & $4.3^{* * *}$ & $1.9^{* *}$ & $4.7^{* * *}$ \\
\hline 86 & Perovskia abrotanoides & Lamiaceae & $\mathrm{L}$ & 89.3 & 100 & $4.3^{* * *}$ & 3.3 & 0 & 0 & 0 & 0 & 31.8 & 35.9 & 24.9 & 43.4 & 24.6 & 40.5 \\
\hline 87 & Petroselinum sativum & Apiaceae & Fr & 96.4 & 96.5 & 2.5 & 2.4 & 0 & 0 & 3.6 & 3.6 & 19.4 & 31.2 & 36.5 & 85 & 28.8 & 62 \\
\hline 88 & Pimpinella anisum & Apiaceae & Fr & $0.0^{* * * *}$ & $0.0^{* * * * * *}$ & & & 0 & 0 & $100^{* * * * * *}$ & $100^{* * * * * *}$ & $1.9^{* * *}$ & $5.8^{* * * *}$ & $0.5^{* *}$ & $4.2 * * *$ & $0.0^{* *}$ & $0.0^{* * *}$ \\
\hline 89 & Pinus eldarica & Pinaceae & $\mathrm{O}$ & 100 & 100 & 2 & 2.3 & 0 & 0 & 0 & 0 & 23.1 & 58.7 & $13.6^{*}$ & 65.7 & 17.2 & 63 \\
\hline 90 & Pinus eldarica & Pinaceae & $\mathrm{L}$ & 100 & 89.3 & 3 & 3 & 0 & $7.2 *$ & 0 & 0 & 66.7 & 71.8 & 81.4 & 79.7 & 75.8 & 68.5 \\
\hline 91 & Pinus eldarica & Pinaceae & $\mathrm{L}$ & 96.4 & 96.5 & 3.4 & 3 & 0 & 0 & 0 & 0 & 44.6 & 32.2 & 53.6 & $25.6^{* *}$ & 48.4 & 27.1 * \\
\hline 92 & Pistacia vera & Anacardiaceae & $\mathrm{Fp}$ & 100 & 100 & 3 & 2.4 & 0 & 0 & 0 & 0 & 47.3 & 51 & 60.2 & 69.2 & 55.2 & 62.2 \\
\hline 93 & Rosmarinus officinalis & Lamiaceae & $\mathrm{L}$ & $35.7^{* *}$ & 89.3 & $5.0 * * * *$ & $4.5^{* * * *}$ & $10.7^{*}$ & 0 & 0 & 0 & $8.8^{* *}$ & 28.1 * & $0.8^{* *}$ & $12.8^{* * *}$ & $1.4^{* *}$ & $16.7^{* *}$ \\
\hline 94 & Rosmarinus officinalis & Lamiaceae & $\mathrm{F}$ & $17.9^{* * *}$ & 89.5 & $5.0 * * * *$ & $4.1^{* * *}$ & 10.7 * & 0 & 0 & 0 & 19.4 & 39.2 & $11.2 * *$ & 45.6 & $2.6^{* *}$ & 38.5 \\
\hline 95 & Ruta graveolens & Rutaceae & $\mathrm{L}$ & $3.6^{* * * *}$ & 14.5 & 4 & 2.5 & $42.9^{* * * * *}$ & ${ }_{* *}^{10.7}$ & $39.3 * *$ & 10.7 & $14.0^{* *}$ & $18.7^{* *}$ & $15.2^{*}$ & $23.0 * *$ & $0.5^{* *}$ & $3.1^{* * *}$ \\
\hline 96 & Salvia nemorosa & Lamiaceae & Ap & 67.9 & 100 & $4.1^{* *}$ & 3.2 & 0 & 0 & 7.2 & 0 & $11.7^{* *}$ & $18.7^{* *}$ & $0.3^{* * *}$ & $11.7^{* * *}$ & $3.2 * *$ & $14.4^{* *}$ \\
\hline 97 & Salvia officinalis & Lamiaceae & A & 96.4 & 100 & $4.2^{* *}$ & $3.4 *$ & 0 & 0 & 0 & 0 & $5.6^{* * *}$ & $14.3^{* * *}$ & $0.5^{* *}$ & $6.7^{* * *}$ & $2.4^{* *}$ & $9.6^{* * *}$ \\
\hline 98 & Salvia syriaca & Lamiaceae & Ap & 67.9 & 78.5 & $4.5^{* * *}$ & 3 & 3.6 & 0 & 3.6 & 3.6 & 26 & 40.2 & 44.6 & 65.9 & 25.4 & 44 \\
\hline 99 & Santolina chamaecyparissus & Asteraceae & $\mathrm{L}, \mathrm{F}$ & 92.9 & 100 & 3 & 2.4 & 0 & 0 & 3.6 & 0 & 24.8 & $31.2 *$ & 27 & 38.8 & 24.3 & 35.8 \\
\hline 100 & Syzygium aromaticum & Myrtaceae & $\mathrm{F}$ & $46.4^{*}$ & 93 & 2.1 & 2.4 & 0 & 3.6 & 21.5 & 0 & $10.6 * *$ & $20.9^{* *}$ & $16.0 *$ & $29.5 * *$ & $6.5^{* *}$ & $24.3 *$ \\
\hline 101 & Tanacetum balsamita & Asteraceae & $\mathrm{L}, \mathrm{F}$ & $3.6^{* * * *}$ & 100 & 5 & 3.3 & 0 & 0 & $25.0 *$ & 0 & $3.7^{* * *}$ & $11.0^{* * *}$ & $0.0^{* * *}$ & $10.2 * * *$ & $0.1 * *$ & $10.5^{* * *}$ \\
\hline 102 & Thuja occidentalis & Cupressaceae & $\mathrm{L}$ & 71.4 & 92.8 & $4.7 * * * *$ & $4.0^{* * *}$ & 0 & 0 & 3.6 & 3.6 & $14.3 * *$ & $24.7 * *$ & $3.1^{* *}$ & $26.2 * *$ & $5.3^{* *}$ & $23.8^{* *}$ \\
\hline 103 & Thuja orientalis & Cupressaceae & $\mathrm{L}$ & 96.4 & 89.3 & 3.1 & 3.2 & 3.6 & 0 & 0 & 3.6 & 21.1 & 32.3 & $16.4^{*}$ & $30.5 *$ & 17.6 & $27.9^{*}$ \\
\hline 104 & Thymus daenensis & Lamiaceae & $\mathrm{L}$ & $0.0^{* * * *}$ & $0.0^{* * * * *}$ & & & 0 & 0 & $100^{* * * * *}$ & $100^{* * * * *}$ & $0.8^{* * *}$ & $5.8^{* * * *}$ & $0.0^{* * *}$ & $0.5^{* * * *}$ & $0.0^{* *}$ & $0.0^{* * *}$ \\
\hline 105 & Thymus transcaspicus & Lamiaceae & $\mathrm{L}$ & $0.0^{* * * *}$ & 10.5 & & $5.0^{* * * * *}$ & 0 & 3.6 & $96.4^{* * * * *}$ & $57.1^{* * * * *}$ & $13.5^{* *}$ & $22.5^{* *}$ & $15.4^{*}$ & $26.7^{* *}$ & $0.0^{* *}$ & $2.7^{* * *}$ \\
\hline 106 & Trachyspermum ammi & Apiaceae & Fr & $3.6^{* * * *}$ & 10.5 & 4 & $3.7^{* *}$ & $67.8^{* * * * *}$ & $\begin{array}{l}39.3 \\
* * * * * *\end{array}$ & 14.3 & $28.6^{* * *}$ & $13.5^{* *}$ & 35.8 & $13.6^{*}$ & 49.6 & $0.5^{* *}$ & $4.7^{* * *}$ \\
\hline 107 & Vitex agnus castus & Verbenaceae & $\mathrm{Fr}$ & 96.4 & 89.5 & 2.6 & 2.4 & 0 & 0 & 3.6 & 0 & 29.7 & 45.3 & 50.5 & 49.9 & 40.9 & 42.9 \\
\hline 108 & Xanthium strumarium & Asteraceae & $\mathrm{L}$ & 64.3 & 96.5 & $4.0^{* *}$ & $3.3 *$ & 0 & 0 & 0 & 0 & 24.1 & 44.4 & $13.3^{* *}$ & 43.5 & $11.2 *$ & 42.3 \\
\hline 109 & Zataria multiflora & Lamiaceae & $\mathrm{L}, \mathrm{F}$ & $0.0 * * * *$ & $39.5^{* * *}$ & & $4.0 * * *$ & 0 & 0 & $100^{* * * * *}$ & $53.6^{* * * * *}$ & $6.7^{* *}$ & $11.1^{* * *}$ & $3.9^{* *}$ & $8.2 * * *$ & $0.0 * *$ & $3.7^{* * *}$ \\
\hline 110 & Zingiber officinale & Zingiberaceae & $\mathrm{Rh}$ & 64.3 & 100 & 3.5 & 2.8 & 7.2 & 0 & 3.6 & 0 & 19.4 & $25.7^{* *}$ & $17.2 *$ & $30.6 *$ & $11.6^{*}$ & $28.7^{*}$ \\
\hline 111 & Ziziphora clinopodioides & Lamiaceae & $\mathrm{L}$ & $7.2^{* * *}$ & 93 & 4.5 & $3.8^{* *}$ & $28.6^{* * * *}$ & 3.6 & 10.7 & 0 & $11.0^{* *}$ & $28.7 *$ & $8.2^{* *}$ & 40.5 & $0.7^{* *}$ & 33.4 \\
\hline 112 & Ziziphora tenuior & Lamiaceae & $\mathrm{L}$ & $0.0^{* * * *}$ & 10.8 & & 3.5 & $71.4^{* * * * *}$ & ${ }_{* * * * * *}^{67.8}$ & $28.6^{*}$ & 7.2 & $9.8^{* *}$ & $19.8^{* *}$ & $14.5^{*}$ & $21.9^{* *}$ & $0.0 * *$ & $2.3^{* * *}$ \\
\hline
\end{tabular}

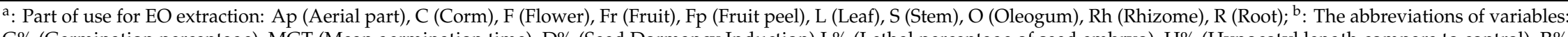
G\% (Germination percentage), MGT (Mean germination time), D\% (Seed Dormancy Induction) L\% (Lethal percentage of seed embryo), H\% (Hypocotyl length compare to control), R\% (Radicle length compare to control), VI (Vigor Index); *: The severity of the inhibitory effect on traits was defined by deviation value at five levels: ${ }^{(* * * *)}$ Mean \pm 2 sd, ${ }^{* * * *)}$ Mean \pm 1.5 sd,

$(* * *)$ Mean $\pm 1 \mathrm{sd},\left({ }^{* *}\right)$ Mean $\pm 0.5 \mathrm{sd}$, and $(*)$ Mean $\pm 0.25 \mathrm{sd}$. 


\subsubsection{Germination Percentage (G\%)}

Most EOs reduced G\% and some had no effect on it. In $1 \mu \mathrm{L}, 36$ EOs were ineffective on this trait and 76 EOs decreased the index compared to the control. At this amount, the highest degree of reduction (less than 22\% compared to control) was observed in 12 EOs of Lamiaceae (Melissa officinalis, Thymus daenensis, Thymus transcaspicus, Origanum vulgare leaf, Ziziphora tenuior, Mentha suaveolens), Geraniaceae (Pelargonium graveolens leaf and flower), Apiaceae (Pimpinella anisum and Trachyspermum ammi), Liliaceae (Allium hirtifolium) and Rutaceae (Ruta graveolens). The inhibitory effects were greater in $3 \mu \mathrm{L}, 89 \mathrm{EOs}$ reduced this trait and $23 \mathrm{EOs}$ were ineffective compared to control. At this amount, the highest degree of reduction (less than 5\% compared to control) was observed in 21 EOs of Lamiaceae (Dracocephalum moldavica and Zataria multiflora in addition to six EOs that were effective at $1 \mu \mathrm{L}$ ), Geraniaceae (as same as the EOs that were effective at $1 \mu \mathrm{L}$ ), Apiaceae (Cuminum cyminum, Bunium persicum, and Ferula foetida in addition to two EOs that were effective at $1 \mu \mathrm{L}$ ), Liliaceae (A. hirtifolium) and Rutaceae (R. graveolens and Citrus aurantifolia leaf), Asteraceae (Artemisia sieberi and Tanacetum balsamita), and Zingiberaceae (Amomum subulatum).

\subsubsection{Mean Germination Time (MGT)}

The EOs also had a significant effect on MGT. The values of this trait were 1.7 to 5 days in $1 \mu \mathrm{L}$, 2 to 5 days in $3 \mu \mathrm{L}$, and 2.1 days in control. In $1 \mu \mathrm{L}, 95$ EOs increased and four EOs decreased this trait compared to control and the rest had no effect. At this amount, the highest increase (more than $224 \%$ compared to control) belonged to four EOs of Lamiaceae (T. transcaspicus and D. moldavica), Asteraceae (A. sieberi), and Zingiberaceae (A. subulatum). At $3 \mu \mathrm{L}, 70 \mathrm{EOs}$ increased and $40 \mathrm{EOs}$ reduced MGT compared to control. At this amount, the highest degree of increase (more than 204\% compared to control) belonged to nine EOs of Lamiaceae (Rosmarinus officinalis leaf and flower, and Perovskia abrotanoides), Asteraceae (A. wilhelmsii, Artemisia aucheri, and Artemisia scoparia), Cupressaceae (Chamaecyparis lawsoniana and Thuja occidentalis), and Apiaceae (Apium graveolens).

\subsubsection{Lethal Percentage of Seed Embryo (L\%)}

The lethal percentage of seed embryo (L\%) was also affected by EO treatment. At $1 \mu \mathrm{L}, 32 \mathrm{EO}$ showed lethal effects. The highest lethality (over $45 \%$ ) belonged to six EOs of Lamiaceae (O. vulgare leaf, T. daenensis, Z. multiflora, and T. transcaspicus), Apiaceae (P. anisum), and Liliaceae (A. hirtifolium). Only T. daenensis and P. anisum EOs were $100 \%$ lethal. At $3 \mu \mathrm{L}, 51 \mathrm{EO}$ showed lethal effects. The highest lethality (over 78\%) belonged to the $13 \mathrm{EOs}$ of the families Lamiaceae (D. moldavica, M. officinalis, and M. suaveolens in addition to four EOs that were effective at $1 \mu \mathrm{L})$, Apiaceae (C. cyminum, F. foetida, and P. anisum), Liliaceae (A. hirtifolium), Geraniaceae (P. graveolens leaf and flower), and Zingiberaceae (A. subulatum). In this amount EOs of F. foetida, P. anisum, M. officinalis, O. vulgare leaf, T. daenensis, Z. multiflora, and A. hirtifolium caused $100 \%$ lethality.

\subsubsection{Seed Dormancy Induction ( $\mathrm{D} \%)$}

Some of the EOs in this experiment induced seed dormancy. The $\mathrm{D} \%$ in the amount of $1 \mu \mathrm{L}$ was $0-67.8 \%$ and in $3 \mu \mathrm{L}$ was $0-71.4 \%$. In $1 \mu \mathrm{L}, 25 \mathrm{EOs}$ induced seed dormancy. The highest degree of seed dormancy induction (more than 22\%) belonged to four EOs of Lamiaceae (Z. tenuior), Apiaceae (T. ammi) and Geraniaceae (P. graveolens leaf and flower). In $3 \mu \mathrm{L}, 35$ EOs showed dormant effects on seed. The highest degree of dormancy induction of seed (over 30\%) belonged to five EOs of Lamiaceae (Z. tenuior and Nepeta cataria), Apiaceae (T. ammi and Anethum graveolens), and Rutaceae (R. graveolens).

\subsection{Allelopathic Effects of Essential Oils on Lettuce Seedling Growth}

Seedlings growth were also significantly affected by treatments (Table 1). 


\subsubsection{Hypocotyl Growth (H\%)}

All EOs decreased hypocotyl length compare to control (H\%). Hypocotyl length was $16.6 \mathrm{~mm}$ in control, $0.8-15.7 \mathrm{~mm}$ in $1 \mu \mathrm{L}$, and $0.1-14.1 \mathrm{~mm}$ in $3 \mu \mathrm{L}$. At $1 \mu \mathrm{L}$ the highest degree of reduction (less than $8 \%$ compared to control) was observed in four EOs of Lamiaceae (O. vulgare flower, P. abrotanoides flower, and T. daenensis) and Apiaceae (P. anisum). At $3 \mu \mathrm{L}$, the highest degree of reduction (less than $6 \%$ compared to control) was observed in $11 \mathrm{EOs}$ from Lamiaceae (D. moldavica, Lavandula angustifolia flower, O. vulgare flower and leaf, T. daenensis, and Salvia officinalis), Apiaceae (P. anisum and A. graveolens), Zingiberaceae (A. subulatum), and Asteraceae (T. balsamita and A. ludoviciana).

\subsubsection{Radicle Growth ( $\mathrm{R} \%)$}

Radicle length compare to control ( $\mathrm{R} \%)$ was also affected by different amounts of the EOs. The radicle length was $26.5 \mathrm{~mm}$ in control, $0.1-28.3 \mathrm{~mm}$ in $1 \mu \mathrm{L}$, and $0-24.9 \mathrm{~mm}$ in $3 \mu \mathrm{L}$. The highest radicle inhibitory degree (less than $3.4 \%$ compared to control) in $1 \mu \mathrm{L}$ was observed in two EOs of Lamiaceae (O. vulgare flower and T. daenensis). The only EO that increased radicle growth at $1 \mu \mathrm{L}$ was coffee EO, which increased $6.9 \%$ this trait compared to control. At $3 \mu \mathrm{L}$, the highest radicle growth inhibition (less than $0.5 \%$ compared to control) belonged to eight EOs of Lamiaceae (L. angustifolia flower, O. vulgare flower and leaf, Salvia nemorosa, and T. daenensis) and Asteraceae (T. balsamita, A. aucheri, and A. deserti). In this amount of EOs T. balsamita, A. aucheri, and A. deserti and T. daenensis were the main radicle growth inhibitors of $100 \%$.

\subsubsection{Vigor Index (VI)}

Vigor index (VI) was also significantly affected by essential oil treatment. The index was 4300 in control, $0-0.4399$ in $1 \mu \mathrm{L}$, and 0-3487 in $3 \mu \mathrm{L}$. At $1 \mu \mathrm{L}$ the highest degree of reduction (less than $12 \%$ compared to control) was observed in $25 \mathrm{EOs}$ of Lamiaceae (D. moldavica, L. angustifolia, O. vulgare flower and leaf, M. officinalis, M. suaveolens, P. abrotanoides flower, S. officinalis, T. transcaspicus, T. daenensis, Z. multiflora, and Z. tenuior), Asteraceae (A. ludoviciana, A. vulgaris flower, A. sieberi, and T. balsamita), Apiaceae (B. persicum, T. ammi, and P. anisum), Geraniaceae (P. graveolens flower and leaf), Liliaceae (A. hirtifolium), Zingiberaceae (A. subulatum), Rutaceae (R. graveolens and C. aurantifolia leaf). At $3 \mu \mathrm{L}$ the highest degree of reduction (less than 10\% compared to control) was observed in 50 EOs of Lamiaceae (Mentha pulegium, N. cataria, N. binaludensis, Mentha longifolia, Mentha piperita, R. officinalis flower and leaf, Ziziphora clinopodioides, S. nemorosa in addition to those were effective in $1 \mu \mathrm{L}$ ), Asteraceae (Achillea filipendulina, Achillea wilhelmsii, A. aucheri, A. deserti, A. scoparia, Chrysanthemum morifolium, in addition to those were effective in $1 \mu \mathrm{L}$ ), Apiaceae (A. graveolens, Coriandrum sativum, C. cyminum, F. foetida, Foeniculum vulgare fruit, Heracleum persicum, in addition to those were effective in $1 \mu \mathrm{L}$ ), Geraniaceae (P. graveolens flower and leaf), Liliaceae (A. hirtifolium), Zingiberaceae (A. subulatum), Rutaceae (R. graveolens and C. aurantifolia leaf), Cupressaceae (Thuja occidentalis), Myrtaceae (Syzygium aromaticum), and Verbenaceae (Lippia citriodora).

\subsection{Headspace Gas Chromatography-Mass Spectrometry (HS-GC-MS)}

Headspace analysis was performed for 16 EOs that inhibit seed germination and seedling growth (Table 2). The results showed that alpha and beta pinene and limonene were among the most common constituents in essential oils, which were observed in seven EOs (alpha pinene $=11-47 \%$, beta pinene $=7-24 \%$, limonene $=11-48 \%)$. Eucalyptol was the most volatile compound in four EOs $(15-39 \%)$. Thujone, camphor, camphene, and borneol were the two most volatile constituents of the two EOs $(21-23 \%, 11-33 \%, 11-23 \%$, and $30-40 \%$, respectively). Other compounds found in headspace analysis were found in only one EO, but some were highly potent, such as carvacrol, which was $91 \%$ of the volatile compounds in $T$. daenensis $\mathrm{EO}$. 
Table 2. The main components of EOs based on Headspace Gas Chromatography-Mass Spectrometry (HS-GC-MS) analysis.

\begin{tabular}{|c|c|c|c|c|c|}
\hline \multirow{2}{*}{$\begin{array}{l}\text { Plant Scientific Name } \\
\text { Amomum subulatum }\end{array}$} & \multirow{2}{*}{$\frac{\text { Part of Use }^{\mathbf{a}}}{\mathrm{Fr}}$} & \multicolumn{4}{|c|}{ Main Components of EOs Based on HS-GC-MS } \\
\hline & & $\begin{array}{c}\text { Dihydrocarveol } \\
(32.1 \%)\end{array}$ & $\beta$-Pinene $(23.1 \%)$ & $\alpha$-Pinene $(18.4 \%)$ & \\
\hline Anethum graveolens & Fr & $\begin{array}{l}\text { 1,2-Diisopropenylcy } \\
\text { clobutane }(74.7 \%)\end{array}$ & & & \\
\hline Artemisia ludoviciana & $\mathrm{L}$ & Borneol (44.2\%) & Camphor $(33.3 \%)$ & Eucalyptol (22.4\%) & \\
\hline Artemisia vulgaris & $\mathrm{L}, \mathrm{F}$ & $\begin{array}{l}\text { D-Limonene } \\
(33.7 \%)\end{array}$ & Thujone $(22.5 \%)$ & $\beta$-Pinene $(14.9 \%)$ & \\
\hline Citrus aurantifolia & $\mathrm{L}$ & $\beta$-Pinene $(20.8 \%)$ & $\begin{array}{c}\text { Linalyl } \\
\text { anthranilate } \\
(20.8 \%)\end{array}$ & Linalol (16.2\%) & $\begin{array}{c}\text { Limonene } \\
(11.2 \%)\end{array}$ \\
\hline Dracocephalum moldavica & Ap & Orthodene (32.6\%) & $\begin{array}{l}\text { Limonene } \\
(20.3 \%)\end{array}$ & $\alpha$-Pinene (17.4\%) & $\begin{array}{l}\text { 4-Carene } \\
(11.3 \%)\end{array}$ \\
\hline Mentha suaveolens & $\mathrm{L}$ & Limonene $(47.9 \%)$ & $\alpha$-Pinene $(16.7 \%)$ & $\beta$-Pinene $(14.0 \%)$ & \\
\hline $\begin{array}{c}\text { Origanum vulgare subsp. } \\
\text { vulgare }\end{array}$ & $\mathrm{L}$ & Carvacrol $(44.4 \%)$ & Thymol (23.0\%) & o-Cymene (18.8\%) & \\
\hline Perovskia abrotanoides & $\mathrm{F}$ & Borneol (30.1\%) & $\begin{array}{l}\text { Eucalyptol } \\
(21.9 \%)\end{array}$ & Camphor (11.1\%) & \\
\hline Pimpinella anisum & Fr & $\begin{array}{c}\text { Trans- anethole } \\
(93.3 \%)\end{array}$ & & & \\
\hline Ruta graveolens & $\mathrm{L}$ & $\begin{array}{c}\beta \text {-Terpinyl acetate } \\
(40.8 \%)\end{array}$ & $\beta$-Pinene $(16.5 \%)$ & $\alpha$-Pinene $(10.7 \%)$ & \\
\hline Tanacetum balsamita & $\mathrm{L}, \mathrm{F}$ & Limonene $(40.2 \%)$ & Thujone (21.0\%) & & \\
\hline Thymus daenensis & $\mathrm{L}$ & Carvacrol (90.9\%) & & & \\
\hline Thymus transcaspicus & $\mathrm{L}$ & Camphene $(23.0 \%)$ & $\alpha$-Pinene (11.0\%) & & \\
\hline Zataria multiflora & $\mathrm{L}, \mathrm{F}$ & o-Cymene (22.7\%) & $\alpha$-Pinene (22.1\%) & Thymol (20.4\%) & \\
\hline Ziziphora tenuior & $\mathrm{L}$ & $\beta$-Pinene $(24.2 \%)$ & $\alpha$-Pinene (21.4\%) & Limonene $(20.6 \%)$ & \\
\hline
\end{tabular}

${ }^{a}$ : Part of use for EO extraction: Ap (Aerial part), C (Corm), F (Flower), Fr (Fruit), Fp (Fruit peel), L (Leaf), S (Stem), $\mathrm{O}$ (Oleogum), Rh (Rhizome), R (Root)

\subsection{Determination of Effective Concentration of EOs}

Determination of effective concentration of EOs on germination inhibitory $\left(\mathrm{IC}_{50}\right)$ and Lethality $\left(\mathrm{LC}_{50}\right)$ was performed in 16 EOs including $P$. graveolens flower and leaf, A. subulatum, C. aurantifolia leaf, R. graveolens, F. foetida, T. ammi, P. anisum, D. moldavica, O. vulgare leaf, M. officinalis, M. suaveolens, T. transcaspicus, T. daenensis, Z. multiflora, and Z. tenuior. According to the results (Table 3 and Figure 4), T. daenensis $\mathrm{EO}$ had the lowest $\mathrm{IC}_{50}\left(\mathrm{IC}_{50}=2.9 \mathrm{ppm}\right)$, indicating that this $\mathrm{EO}$ has the highest allelopathic potential in this regard. Thereafter, the lowest effective concentrations were related to P. anisum, Z. multiflora, O. vulgare and Z. tenuior $\mathrm{EOs}\left(\mathrm{IC}_{50}=7.5 \mathrm{ppm}, \mathrm{IC}_{50}=7.9 \mathrm{ppm}\right.$, $\mathrm{IC}_{50}=11.4 \mathrm{ppm}$ and $\mathrm{IC}_{50}=13.7 \mathrm{ppm}$, respectively). T. daenensis $\mathrm{EO}$ was the most lethal EO with the lowest lethal concentration $\left(\mathrm{LC}_{50}=7.2 \mathrm{ppm}\right)$. Subsequently, the EOs of O. vulgare, Z. multiflora, P. anisum, $T$. transcaspicus had the lowest lethal concentrations $\left(\mathrm{LC}_{50}=16.2 \mathrm{ppm}, \mathrm{LC}_{50}=21.6 \mathrm{ppm}\right.$, $\mathrm{LC}_{50}=22.2 \mathrm{ppm}$, and $\mathrm{LC}_{50}=23.3 \mathrm{ppm}$, respectively).

Determination of effective concentration of EOs on hypocotyl inhibition (HIC), radicle inhibition (RIC), and seedling inhibition (SIC) was performed in 20 EOs including P. graveolens flower and leaf, A. hirtifolium, A. subulatum, A. graveolens, P. anisum, A. aucheri, A. ludoviciana, A. sieberi, T. balsamita, L. angustifolia flower, $M$. suaveolens, $O$. vulgare leaf and flower, $P$. abrotanoides flower, $R$. officinalis leaf, S. nemorosa, S. officinalis, Z. multiflora, and Z. tenuior was done on radicle (Table 4 and Figure 5). Results showed the strongest $\mathrm{EO}$ with the least $\mathrm{IC}_{50}$ of hypocotyl, radicle, and seedling growth was $O$. vulgare flower $\mathrm{EO}\left(\mathrm{HIC}_{50}=12.9 \mathrm{ppm}, \mathrm{RIC}_{50}=9.6 \mathrm{ppm}, \mathrm{SIC}_{50}=10.5 \mathrm{ppm}\right)$. Afterwards, A. hirtifolium, L. angustifolia flower, and P. graveolnes leaf and flower EOs were the most effective on hypocotyl inhibition $\left(\mathrm{HIC}_{50}=18.1 \mathrm{ppm}, \mathrm{HIC}_{50}=22.8 \mathrm{ppm}, \mathrm{HIC}_{50}=27.8 \mathrm{ppm}\right.$, and $\mathrm{HIC}_{50}=30.1 \mathrm{ppm}$, respectively); A. ludoviciana, P. abrotanoides flower, S. officinalis, and P. anisum had the highest effective on radicle growth $\left(\mathrm{RIC}_{50}=18.3 \mathrm{ppm}, \mathrm{RIC}_{50}=19.1 \mathrm{ppm}, \mathrm{RIC}_{50}=23.4 \mathrm{ppm}\right.$, and $\mathrm{RIC}_{50}=27.8 \mathrm{ppm}$, respectively); and P. abrotanoides flower, A. ludoviciana, L. angustifolia flower, and S. officinalis EOs 
showed the most effect on seedling growth $\left(\mathrm{SIC}_{50}=20.7 \mathrm{ppm}, \mathrm{SIC}_{50}=24.9 \mathrm{ppm}, \mathrm{SIC}_{50}=28.7 \mathrm{ppm}\right.$, and $\mathrm{SIC}_{50}=29.1 \mathrm{ppm}$, respectively).

Table 3. The effective concentration of EOs on seed germination inhibition and embryo lethal by cotton swab method.

\begin{tabular}{|c|c|c|c|c|c|c|c|}
\hline \multirow{3}{*}{ Plant Scientific Name } & \multirow{3}{*}{$\begin{array}{c}\text { Part of } \\
\text { Use }^{\text {a }}\end{array}$} & \multicolumn{3}{|c|}{ Seed Germination Inhibition } & \multicolumn{3}{|c|}{ Embryo Lethal Effect } \\
\hline & & $\mathrm{IC}_{25}$ & $\mathrm{IC}_{50}$ & $\mathrm{IC}_{90}$ & $\mathrm{LC}_{25}$ & $\mathrm{LC}_{50}$ & $\mathrm{LC}_{90}$ \\
\hline & & \multicolumn{6}{|c|}{ ppm } \\
\hline Amomum subulatum & Fr & 33.1 & 50.0 & 114 & 115 & 137 & 196 \\
\hline Citrus aurantifolia & $\mathrm{L}$ & 9.0 & 20.9 & 114 & 23.2 & 64.9 & 510 \\
\hline Dracocephalum moldavica & Ap & 13.3 & 21.0 & 52.3 & 69.4 & 126 & 414 \\
\hline Ferula foetida & $\mathrm{O}$ & 88.6 & 115 & 193.0 & 119 & 138 & 183 \\
\hline Melissa officinalis & $\mathrm{L}$ & 36.3 & 48.0 & 83.8 & 64.9 & 102 & 248 \\
\hline Mentha suaveolens & $\mathrm{L}$ & 22.3 & 32.0 & 66.0 & 57.6 & 93.2 & 244 \\
\hline Origanum vulgare subsp. vulgare & $\mathrm{L}$ & 8.0 & 11.4 & 23.4 & 12.2 & 16.2 & 28.5 \\
\hline Pelargonium graveolnes & $\mathrm{L}$ & 15.8 & 26.3 & 73.1 & 34.8 & 64.9 & 226 \\
\hline Pelargonium graveolnes & $\mathrm{L}, \mathrm{F}$ & 20.8 & 30.0 & 63.0 & 66.7 & 116 & 351 \\
\hline Pimpinella anisum & Fr & 7.0 & 7.5 & 8.5 & 9.4 & 22.9 & 136 \\
\hline Ruta graveolens & $\mathrm{L}$ & 31.8 & 45.6 & 93.7 & 116 & 340 & 2916 \\
\hline Thymus daenensis & $\mathrm{L}$ & 1.8 & 2.9 & 8.0 & 3.8 & 7.2 & 25.3 \\
\hline Thymus transcaspicus & $\mathrm{L}$ & 12.9 & 17.3 & 30.8 & 18.3 & 23.1 & 36.7 \\
\hline Trachyspermum ammi & Fr & 18.0 & 25.0 & 48.3 & 52.6 & 153 & 1294 \\
\hline Zataria multiflora & $\mathrm{L}, \mathrm{F}$ & 5.1 & 7.9 & 18.4 & 6.9 & 21.6 & 213 \\
\hline Ziziphora tenuior & $\mathrm{L}$ & 8.6 & 13.7 & 35.4 & 30.3 & 49.2 & 130 \\
\hline
\end{tabular}

a: Part of use for EO extraction: Ap (Aerial part), C (Corm), F (Flower), Fr (Fruit), Fp (Fruit peel), L (Leaf), S (Stem),

$\mathrm{O}$ (Oleogum), Rh (Rhizome), R (Root)

Table 4. The effective concentration of EOs on radicle, hypocotyl, and seedling inhibition by cotton swab method.

\begin{tabular}{|c|c|c|c|c|c|c|c|c|c|c|}
\hline \multirow{3}{*}{ Plant Scientific Name } & \multirow{3}{*}{$\begin{array}{c}\text { Part of } \\
\text { Use }^{a}\end{array}$} & \multicolumn{3}{|c|}{ Hypocotyl Inhibition } & \multicolumn{3}{|c|}{ Radicle Inhibition } & \multicolumn{3}{|c|}{ Seedling Inhibition } \\
\hline & & $\mathrm{IC}_{25}$ & $\mathrm{IC}_{50}$ & $\mathrm{IC}_{90}$ & $\mathrm{IC}_{25}$ & $\mathrm{IC}_{50}$ & $\mathrm{IC}_{90}$ & $\mathrm{IC}_{25}$ & $\mathrm{IC}_{50}$ & $\mathrm{IC}_{90}$ \\
\hline & & \multicolumn{9}{|c|}{ ppm } \\
\hline Allium hirtifolium & $\mathrm{C}$ & 3.4 & 18.1 & 512 & 34.9 & 62.2 & 197 & 18.9 & 46.2 & 278 \\
\hline Amomum subulatum & $\mathrm{Fr}$ & 44.6 & 69.3 & 168 & 50.8 & 70.2 & 134 & 48.8 & 70.7 & 148 \\
\hline Anethum graveolens & Fr & 20.6 & 46.7 & 238 & 30.4 & 64.4 & 289 & 24.8 & 56.2 & 287 \\
\hline Artemisia aucheri & $\mathrm{L}$ & 39.7 & 79.7 & 321 & 63.8 & 73.0 & 95.6 & 50.8 & 72.3 & 147 \\
\hline Artemisia ludoviciana & $\mathrm{L}$ & 15.4 & 37.8 & 227 & 10.5 & 18.3 & 54.8 & 11.6 & 24.9 & 116 \\
\hline Artemisia vulgaris & $\mathrm{L}, \mathrm{F}$ & 15.2 & 59.1 & 899 & 33.5 & 79.4 & 448 & 24.3 & 71.3 & 612 \\
\hline Artemisia sieberi & $\mathrm{L}, \mathrm{S}$ & 11.3 & 38.4 & 446 & 10.5 & 28.4 & 209 & 10.8 & 31.6 & 270 \\
\hline Lavandula angustifolia & $\mathrm{F}$ & 9.9 & 27.8 & 221 & 17.6 & 29.3 & 80.5 & 14.5 & 28.7 & 113 \\
\hline Mentha suaveolens & $\mathrm{L}$ & 16.0 & 31.5 & 122 & 19.0 & 32.8 & 97.0 & 17.9 & 32.2 & 105 \\
\hline Origanum vulgare subsp. viridi & $\mathrm{F}$ & 5.4 & 12.9 & 73.9 & 6.2 & 9.6 & 23.2 & 6.0 & 10.5 & 31.6 \\
\hline Origanum vulgare subsp. vulgar & $\mathrm{L}$ & 10.3 & 36.3 & 450 & 18.2 & 45.0 & 276 & 15.2 & 42.1 & 322 \\
\hline Pelargonium graveolnes & $\mathrm{L}, \mathrm{F}$ & 7.4 & 30.1 & 503 & 9.8 & 54.5 & 1693 & 8.3 & 42.2 & 1093 \\
\hline Perovskia abrotanoides & $\mathrm{F}$ & 8.1 & 22.8 & 182 & 13.5 & 19.1 & 38.4 & 12.8 & 20.7 & 54.8 \\
\hline Pimpinella anisum & $\mathrm{Fr}$ & 16.6 & 34.3 & 147 & 14.5 & 27.8 & 103 & 18.9 & 34.1 & 111 \\
\hline Rosmarinus officinalis & $\mathrm{L}$ & 49.6 & 90.4 & 300 & 63.1 & 104 & 279 & 57.9 & 98.7 & 287 \\
\hline Salvia nemorosa & Ap & 33.8 & 67.9 & 275 & 54.6 & 74.3 & 138 & 51.3 & 74.1 & 155 \\
\hline Salvia officinalis & $\mathrm{Ap}$ & 16.1 & 44.6 & 342 & 10.2 & 23.4 & 124 & 11.2 & 29.1 & 196 \\
\hline Tanacetum balsamita & $\mathrm{L}, \mathrm{F}$ & 16.7 & 44.3 & 313 & 24.6 & 56.6 & 300 & 20.8 & 51.3 & 313 \\
\hline Thymus daenensis & $\mathrm{L}$ & 15.1 & 30.7 & 127 & 25.1 & 45.9 & 153 & 22.1 & 40.9 & 140 \\
\hline Zataria multiflora & $\mathrm{L}, \mathrm{F}$ & 26.0 & 50.6 & 193 & 12.7 & 33.8 & 238 & 17.3 & 40.5 & 221 \\
\hline
\end{tabular}

a: Part of use for EO extraction: Ap (Aerial part), C (Corm), F (Flower), Fr (Fruit), Fp (Fruit peel), L (Leaf), S (Stem), $\mathrm{O}$ (Oleogum), Rh (Rhizome), R (Root). 


\subsection{Allelopathic Interaction Effect of EOs}

Based on the results of screening experiments, the interaction effect on seed germination was evaluated in eight EOs. Also, this effect was studied on root and hypocotyl growth separately in six EOs.

\subsubsection{Simplified Modified Dilution Check-Board Technique (SMCT)}

Results of SMCT and combination effect (CE) showed different states of the synergistic and antagonistic effect. In the combination of essential oils regarding germination inhibition, most of the combinations resulted in antagonistic interaction (Table 5). The highest antagonistic effect was observed in the combination of $R$. graveolens $+D$. moldavica $(C E=0.56)$ followed by the $R$. graveolens + T. transcaspicus, and C. aurantifolia $+T$. daenensis $(\mathrm{CE}=0.59$ and $\mathrm{CE}=0.66$, respectively). The synergistic effect was observed only in five combinations, the strongest of which was the combination of A. subulatum $+M$. suaveolens $(\mathrm{CE}=1.25)$. There were also different combination effects of essential oil on hypocotyl growth inhibition. Of the 15 compounds, five had additive interaction, 6 had synergistic effects, and 4 had antagonistic effects (Table 5). The most synergistic interaction was related to P. abrotanoides $+T$. daenensis $(\mathrm{CE}=1.25)$, and the highest antagonistic effect was observed in A. subulatum $+T$. balsamita $(\mathrm{CE}=0.72)$. The effects of essential oil combination on radicle growth inhibition were also different. Most of the combinations showed additive interaction. Among 15 combinations, 4 showed synergistic effect, seven showed additive and 4 other showed antagonistic interaction (Table 5). The highest synergistic effect was observed in A. vulgaris + M. suaveolens $(\mathrm{CE}=1.22)$, and the strongest antagonistic interaction was in A. subulatum + P. abrotanoides $(\mathrm{CE}=0.68)$. 


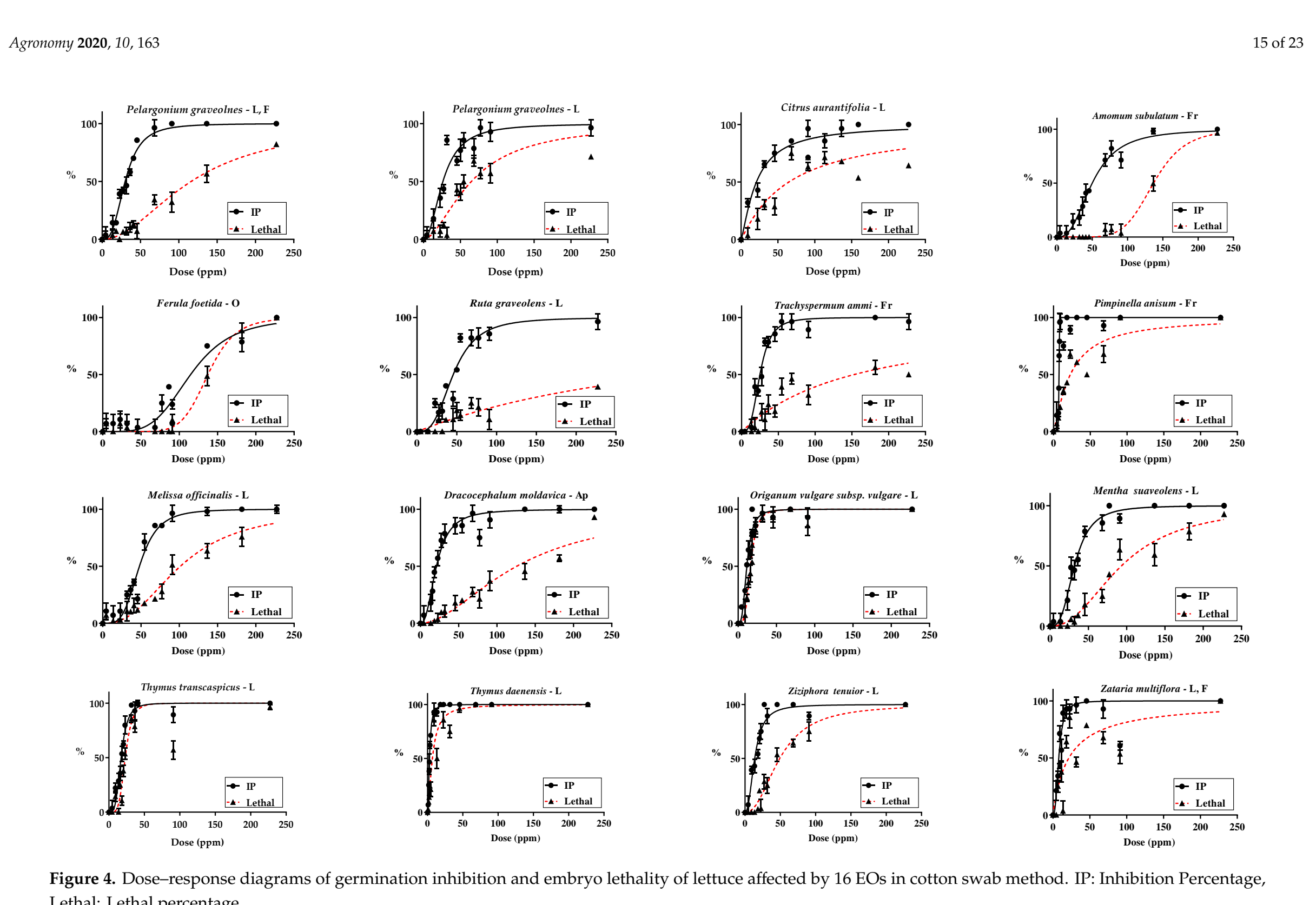



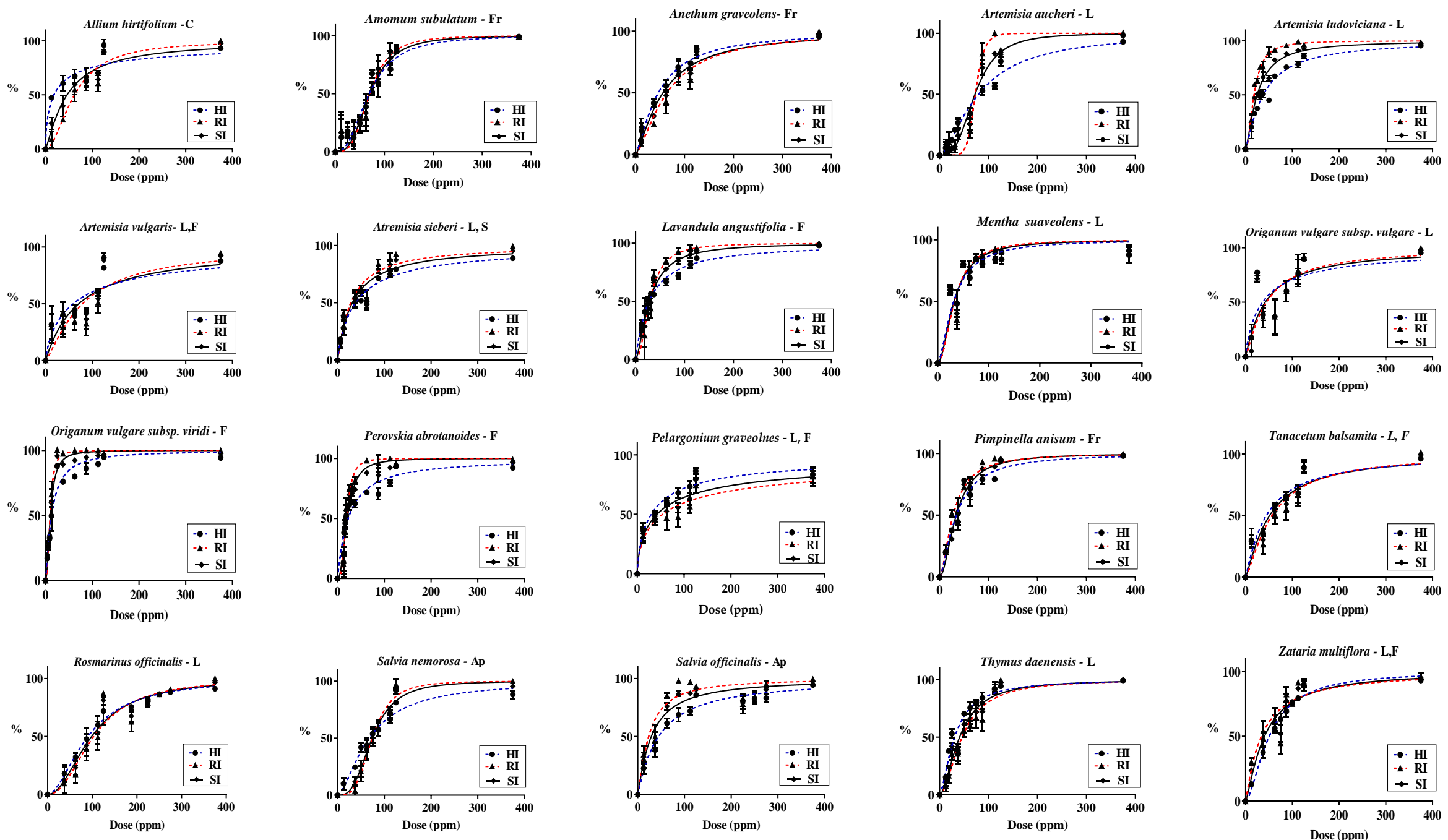

Figure 5. Dose-response diagrams of lettuce seedling growth affected by 20 EOs in cotton swab method. HI: Hypocotyl Inhibition, RI: Radicle Inhibition, SI: Seedling Inhibition. 
Table 5. Allelopathic effects of essential oils mixtures on seed germination, hypocotyl and radicle growth of lettuce based on simplified modified dilution check-board technique (SMCT) by cotton swab method.

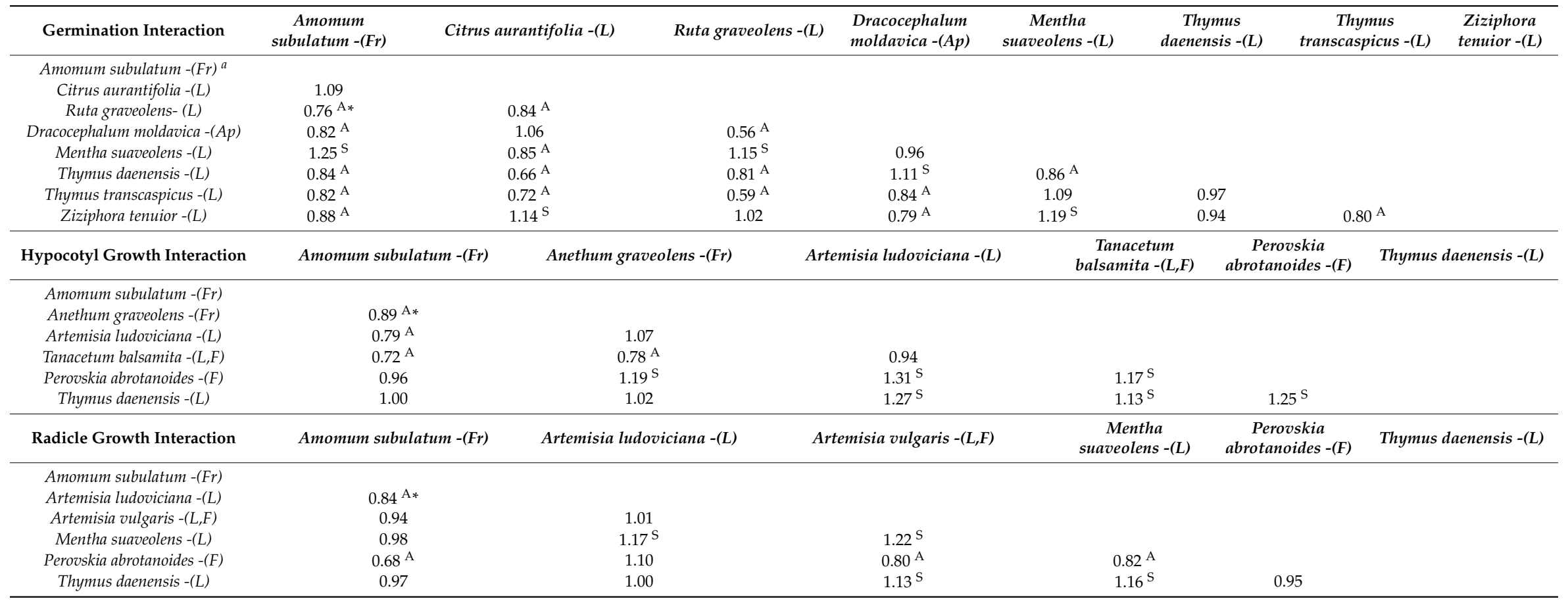

a: Part of use for EO extraction: Ap (Aerial part), C (Corm), F (Flower), Fr (Fruit), Fp (Fruit peel), L (Leaf), S (Stem), O (Oleogum), Rh (Rhizome), R (Root); *: The letters on the numbers indicate the state of interaction between the essential oils: $\mathrm{S}$ : synergistic effect $(\mathrm{CE}>1.1)$, A: antagonistic effect $(\mathrm{CE}<0.9)$. 


\subsubsection{Isobologram Curves}

Isobologram curves showed different type of allelopathic interaction of EOs and they appropriately confirm the SMCT result (Figures S1-S3). In the isobologram curves of germination inhibition (Figure S1), eight EOs showed synergistic effects, 16 EOs had antagonistic effects and four others showed additive effects. Among the EOs, M. suaveolens had the most synergistic effect with other EOs (four synergistic effects), while A. subulatum, R. graveolens and T. transcaspicus EOs had the most antagonistic effects with the other EOs (five antagonistic effects). In the hypocotyl inhibitory isobologram (Figure S2), four EOs indicating antagonistic status, seven curves indicating synergistic status between EOs, and four other curves indicating additive effects. A. subulatum showed the highest number of antagonistic effects with the other EOs (three antagonistic effects), so that black cardamom did not have synergistic effect with any other EOs. P. abrotanoides and T. daenensis had the highest number of synergistic effects in combination with other EOs (four and three synergistic effects, respectively), so that they showed no antagonistic effect with any other EOs. And finally, in the isobologram curves of radicle growth inhibition (Figure S3) four curves showed antagonistic effects between EOs, five curves indicating synergistic effects and six other curves indicating additive effects. The highest number of antagonistic effects was related to P. abrotanoides EO (three antagonistic effects) and M. suaveolens EO had the highest number of synergistic effects with other EOs (three synergistic effects). In this regard, black cardamom EO had no synergistic effect, and T. daenensis EO showed no antagonistic effect.

\section{Discussion}

The results of this experiment confirm, in many cases, other researchers' findings on the allelopathic effects of EOs and their compounds on seed germination. Monoterpenes can affect the germination of seeds at very low concentrations [25]. The EOs of T. daenensis and P. anisum were among the strongest germination inhibitors in this experiment. The potent microbicidal effects of these species, as well as their antimicrobial, inhibitory or stimulatory effect on enzymatic activity, toxic effects of estragol have been reported in previous studies [26-29]. Phytotoxic effects of T. daenensis EO have been reported in other allelopathic studies. The germination of some weed species has been showed to be strongly inhibited by this species EO [30], so that complete germination inhibition of Avena fatua occurred at $600 \mu \mathrm{L} / \mathrm{L}$ and complete germination inhibition of Amaranthus retroflexus and Datura stramonium occurred at $800 \mu \mathrm{L} / \mathrm{L}$. Antibacterial effects on E. coli using this EO, have also been reported [28], achieving complete bacterial death within five minutes. These results seem to be caused by thymol, carvacrol, and carvone which are the compounds of $T$. daenensis EO that showed highest inhibitory effect on seed germination [31].

The insecticidal effects of $P$. anisum and P. graveolens, especially on larvae killing and oviposition-deterrent, are already known [32,33], as well as their fumigant-pesticide effect [34,35]. In our experiment, these species' EOs showed strong inhibitory and phytotoxic effects. According to Dhima et al. [36], P. anisum had significant inhibitory effect on germination and seedling of Echinochloa crus-galli, although Azirak and Karaman [31] observed effectiveness against seed germination only at high concentration, and not at low ones. Trans-anethole, the dominant constituent of P. anisum EO, is a type of phenylpropanoids that is toxic for some insects [37]. Since this compound was very high in headspace analysis of $P$. anisum EO (90\%), it is most likely to be responsible for inhibition of the seed germination. Despite this, in another study, trans-anethole had no significant effect on seed germination inhibition [38]. The differences in the results may be, in part, due to the use of different plant species in the tests: and it should be noted that lettuce seed is more sensitive to allelochemicals. The way EO was applied in each study, fumigation in ours vs soaking in the others, might have also explain the differences in the results. Concerning the allelopathic effects of O. vulgare, its EO has potent antifungal effects on a wide range of fungi that have been attributed to thymol and carvacrol, among others [39]. These compounds also had germination inhibitory effects on some plant species, whereas p-cymene showed no phytotoxic effect [40]. But Elshafie et al. [41] saw moderate phytotoxic effects of $O$. vulgare EO. According to other studies, Z. multiflora significantly reduced germination rate, 
seedling length, root and shoot weight [42]. Allelopathic effects of leaves of this species on sandwich method have also been reported [43]. The antifungal effects of $Z$. multiflora $\mathrm{EO}$ were also investigated and showed complete inhibition of fungi growth [44]. Allelopathic studies of other researchers have reported phytotoxic effects of $P$. abrotanoides [11,43]. Its flower was one of the most effective inhibitors of lettuce seedling growth in sandwich method [43], and in dish pack method, it had an inhibitory effect of more than $80 \%$ on root growth, which, according to the experimental method, is due to its volatile compounds [11]. For other plants, many herbaceous species of Artemisia contain active compounds that have allelopathic and antifungal effects, and their allelochemicals are mainly volatile ethers and alkaloids. The allelopathic effect of A. ludoviciana showed inhibitory effects on plant growth [45]. The EO of this plant also had antibacterial effects and at a concentration of $10 \mu \mathrm{g} / \mathrm{mL}$ inhibited the growth of some bacteria [46].

Among major volatile compounds resulting from HS-GC-MS, alpha and beta pinene, which were dominant compound in most EOs, are oxygenated bicyclic monoterpenes, which are insoluble in water and highly volatile, and their allelopathic effects on the environment and insect repellent effects have been reported $[47,48]$. Other compounds, like limonene, an aliphatic and cyclic monoterpene, also present phytotoxic effects [49]. Eucalyptol or 1,8-cineole is an ether cyclic monoterpene that has insecticidal and insect repellent effects [50,51], as well as strong growth inhibitory effects on plants, affecting the activity of the asparagine synthase enzyme, thus being one of the most important allelochemical constituents [52]. The toxicity and lethal effects of eucalyptol on rats have been tested and, although it has a relatively high lethal dose $\left(\mathrm{LD}_{50}=2.48 \mathrm{~g} / \mathrm{kg} \mathrm{bw}, \mathrm{rat}\right)$, its long-term effects on living cells can be extremely damaging and should be carefully considered [53] before using it against weeds. Borneol is an alcoholic monoterpene with reports of its inhibitory effects on Schizachyrium scoparium germination ( $8 \%$ ) and radicle growth (47\%), whereas it did not affect Leptochloa dubia germination and seedling growth [54]. Except for O. vulgare and P. abrotanoides, which inhibited the growth of both radicle and hypocotyl, the other EOs did not have the same inhibitory effects on these two seedling parts. Allium hirtifolium, L. angustifolia and P. graveolens had the greatest effect on decreasing hypocotyl growth, while A. ludoviciana, S. officinalis, and P. anisum were more effective in inhibiting radicle growth. This difference is probably due to the different role of the EOs in disrupting hormonal balance, and the effect of their compounds on growth regulators biosynthetic pathways.

Another notable point in this experiment was the different effect of the EO of different parts of a plant. In 11 plants, the EOs were extracted from two different parts of them, which in some cases had different effects. About flowers and leaves EO, in P. abrotanoides, A. vulgaris, and L. angustifolia, the allopathic effect of flower EO was greater than that of leaf, in O. vulgare the effect of the leaf EO was more than the flower, and there was no difference in geranium and rosemary in this regard. On the effect of leaf and fruit EO on germination, in C. aurantifolia, F. vulgare and Cupressus arizonica the inhibitory effect of leaf oil was greater than that of fruit, and in Juniperus horizontalis there was no difference. Regarding their differences in inhibition of seedling growth, in Juniperus horizontalis and C. arizonica the inhibitory effect of leaf EO was greater than fruit, in F. vulgare the fruit EO effect was more than leaf and in C. aurantifolia there was no difference. Regarding the different effects of Pinus eldarica leaf EO with its oleogum EO, the former had more inhibitory effects on germination than the later.

In this study, synergistic, additive and even antagonistic effects between plant EOs were observed. Synergistic effects between the EOs of some species of Apiaceae family such as Coriander and Cumin have been reported for antibacterial and antioxidant activity [16]. In another study, antifungal synergistic effects were observed between the EO of marjoram and thyme, peppermint and tea tree, and thyme and cinnamon [55]. Antimicrobial synergistic effects between EO components such as eugenol and linalool, eugenol and menthol, and carvacrol and thymol have also been reported [18]. 


\section{Conclusions}

This study represents new results in the allelopathy database. The results showed significant inhibitory effects of essential oils even at $1 \mu \mathrm{L}$ concentration on the studied traits in gaseous phase. $P$. graveolens, P. anisum and T. daenensis EOs had the greatest inhibitory effect on germination; A. subulatum, A. sieberi, D. moldavica and T. transcaspicus EOs had the greatest effect on germination delay; P. anisum and T. daenensis EOs had the greatest effect on seed embryo lethality. P. anisum, O. vulgare, P. abrotanoides and T. daenensis EOs caused the greatest inhibition of seedling growth. In addition, the results of allelopathic interaction of the EOs showed that there were different antagonistic, synergistic, and additive interaction in their combination. M. suaveolens in inhibition of germination and radicle growth, and P. abrotanoides in inhibition of hypocotyl growth, showed the highest synergistic ability in combination with other EOs. A. subulatum, R. graveolens, and T. transcaspicus EOs had the highest antagonistic ability in combination with others. The interaction effects between the EOs can be of great interest in plant-based herbicides production. Such effects have a particular role in determining the appropriate dose so that synergistic effects can more effectively prevent germination and weed growth and reduce herbicide consumption.

Supplementary Materials: The following are available online at http://www.mdpi.com/2073-4395/10/2/163/s1, Figure S1: Isobologram curves of allelopathic interaction effects of EOs on lettuce seed germination inhibition by cotton swab method, Figure S2: Isobologram curves of allelopathic interaction effects of EOs on lettuce hypocotyl growth inhibition by cotton swab method, Figure S3: Isobologram curves of allelopathic interaction effects of EOs on lettuce radicle growth inhibition by cotton swab method, Table S1: Analysis of variance of variables studied in the experiment to investigate the effects of 112 essential oils concentrations on seed germination and seedling growth of lettuce.

Author Contributions: Conceptualization, M.A. and Y.F.; methodology, M.A. and Y.F.; validation, M.A., Y.F. and S.M.; formal analysis, S.M.; investigation, S.M.; resources, S.M. and M.A.; data curation, S.M.; writing - original draft preparation, S.M.; writing-review and editing, M.A.; visualization, S.M.; supervision, M.A.; project administration, S.M.; funding acquisition, M.A and Y.F. All authors read and confirm the manuscript. All authors have read and agreed to the published version of the manuscript.

Funding: This research received funding from the Ministry of Education, Culture, Sports, Science, and Technology (MEXT), Japan. This work was also partly supported by JST CREST Grant Number JPMJCR17O2 and JSPS KAKENHI Grant Number 26304024. "This work was also partly supported by Ferdowsi University of Mashhad under grant number: 38489."

Conflicts of Interest: The authors declare no conflict of interest.

\section{References}

1. Batish, D.R.; Arora, K.; Singh, H.P.; Kohli, R.K. Potential Utilization of Dried Powder of Tagetes minuta as a Natural Herbicide for Managing Rice Weeds. Crop Prot. 2007, 26, 566-571. [CrossRef]

2. Batish, D.R.; Kaur, M.; Singh, H.P.; Kohli, R.K. Phytotoxicity of a Medicinal Plant, Anisomeles indica, against Phalaris minor and Its Potential Use as Natural Herbicide in Wheat Fields. Crop Prot. 2007, 26, 948-952. [CrossRef]

3. Kordali, S.; Cakir, A.; Akcin, T.A.; Mete, E.; Akcin, A.; Aydin, T.; Kilic, H. Antifungal and Herbicidal Properties of Essential Oils and N-Hexane Extracts of Achillea gypsicola Hub-Mor. and Achillea biebersteinii Afan. (Asteraceae). Ind. Crops Prod. 2009, 29, 562-570. [CrossRef]

4. Heap, I.; Peterson, M.; Horak, M. International Survey of Herbicide Resistant Weeds. Available online: http://www.weedscience.org (accessed on 16 September 2019).

5. Jabran, K. Allelopathy: Introduction and Concepts; Springer: Cham, Switzerland, 2017; pp. 1-12. [CrossRef]

6. Rice, E.L. Allelopathy; Academic Press: Orlando, FL, USA, 1984.

7. Zimdahl, R.L. Fundamentals of Weed Science, 5th ed.; Academic Press: San Diego, CA, USA, 2018.

8. Journet, A.R.P.; Etherington, J.R. Plant Physiological Ecology. Bryologist 2006, 82, 508. [CrossRef]

9. Azizi, M.; Fujii, Y. Allelopathic Effect of Some Medicinal Plant Substances on Seed Germination of Amaranthus retroflexus and Portulaca oleraceae. Acta Hortic. 2006, 699, 61-67. [CrossRef] 
10. Azizi, M.; Amini, S.; Joharchi, M.R.; Oroojalian, F.; Baghestani, Z. Genetic Resources for Allelopathic and Medicinal Plants from Traditional Persian Experience. In Proceedings of the MARCO Symposium (Challenges for Agro-Environmental Research in Monsoon Asia), Tsukuba, Japan, 5-7 October 2009.

11. Amini, S.; Azizi, M.; Joharchi, M.R.; Shafei, M.N.; Moradinezhad, F.; Fujii, Y. Determination of Allelopathic Potential in Some Medicinal and Wild Plant Species of Iran by Dish Pack Method. Theor. Exp. Plant Physiol. 2014, 26, 189-199. [CrossRef]

12. Inderijt. Soil Microorganisms: An Important Determinant of Allelopathic Activity. Plant Soil 2005, 274, 227-236. [CrossRef]

13. Azizi, M.; Farzad, S.; Jafarpour, B.; Rastegar, M.F.; Jahanbakhsh, V. Inhibitory Effect of Some Medicinal Plants' Essential Oils on Postharvest Fungal Disease of Citrus Fruits. Acta Hortic. 2008, 768, 279-286. [CrossRef]

14. Oroojalian, F.; Kasra-Kermanshahi, R.; Azizi, M.; Bassami, M.R. Phytochemical Composition of the Essential Oils from Three Apiaceae Species and Their Antibacterial Effects on Food-Borne Pathogens. Food Chem. 2010, 120, 765-770. [CrossRef]

15. Mardani, H.; Maninang, J.; Appiah, K.S.; Oikawa, Y.; Azizi, M.; Fujii, Y. Evaluation of Biological Response of Lettuce (Lactuca sativa 1.) and Weeds to Safranal Allelochemical of Saffron (Crocus sativus) by Using Static Exposure Method. Molecules 2019, 24, 1788. [CrossRef] [PubMed]

16. Bag, A.; Chattopadhyay, R.R. Evaluation of Synergistic Antibacterial and Antioxidant Efficacy of Essential Oils of Spices and Herbs in Combination. PLoS ONE 2015, 10, e0131321. [CrossRef] [PubMed]

17. Oroojalian, F.; Orafaee, H.; Azizi, M. Synergistic Antibaterial Activity of Medicinal Plants Essential Oils with Biogenic Silver Nanoparticles. Nanomed. J. 2017, 4, 237-244. [CrossRef]

18. Chouhan, S.; Sharma, K.; Guleria, S. Antimicrobial Activity of Some Essential Oils—Present Status and Future Perspectives. Medicines 2017, 4, 58. [CrossRef] [PubMed]

19. Alexa, E.; Sumalan, R.M.; Danciu, C.; Obistioiu, D.; Negrea, M.; Poiana, M.A.; Rus, C.; Radulov, I.; Pop, G.; Dehelean, C. Synergistic Antifungal, Allelopatic and Anti-Proliferative Potential of Salvia officinalis L., and Thymus vulgaris L. Essential Oils. Molecules 2018, 23, 185. [CrossRef] [PubMed]

20. Foy, C.L. Understanding the Role of Allelopathy in Weed Interference and Declining Plant Diversity 1. Weed Technol. 2001, 15, 873-878. [CrossRef]

21. Council of Europe. European Directorate for the Quality of Medicines \& HealthCare. In European Pharmacopoeia, 6th ed.; Council of Europe (COE): Strasbourg, France, 2007.

22. Mishyna, M.; Laman, N.; Prokhorov, V.; Maninang, J.S.; Fujii, Y. Identification of Octanal as Plant Growth Inhibitory Volatile Compound Released from Heracleum sosnowskyi Fruit. NPC Nat. Prod. Commun. 2015, 10, 771-774. [CrossRef] [PubMed]

23. Fetouh, M.I.; Hassan, F.A. Seed Germination Criteria and Seedling Characteristics of Magnolia grandiflora L. Trees after Cold Stratification Treatments. Int. J. Curr. Microbiol. Appl. Sci. 2014, 3, 235-241.

24. Leist, N.; Krämer, S.; Jonitz, A. ISTA Working Sheets on Tetrazolium Testing; The International Seed Testing Association (ISTA): Basserdorf, Switzerland, 2003.

25. Dudai, N.; Larkov, O.; Putievsky, E.; Lerner, H.R.; Ravid, U.; Lewinsohn, E.; Mayer, A.M. Biotransformation of Constituents of Essential Oils by Germinating Wheat Seed. Phytochemistry 2000, 55, 375-382. [CrossRef]

26. Sihoglu Tepe, A.; Tepe, B. Traditional Use, Biological Activity Potential and Toxicity of Pimpinella Species. Ind. Crops Prod. 2015, 69, 153-166. [CrossRef]

27. Khalil, N.; Ashour, M.; Fikry, S.; Singab, A.N.; Salama, O. Chemical Composition and Antimicrobial Activity of the Essential Oils of Selected Apiaceous Fruits. Future J. Pharm. Sci. 2018, 4, 88-92. [CrossRef]

28. Moghimi, R.; Ghaderi, L.; Rafati, H.; Aliahmadi, A.; Mcclements, D.J. Superior Antibacterial Activity of Nanoemulsion of Thymus daenensis Essential Oil against E. coli. Food Chem. 2016, 194, 410-415. [CrossRef] [PubMed]

29. Sharma, P.K.; Raina, A.P.; Dureja, P. Evaluation of the Antifungal and Phytotoxic Effects of Various Essential Oils against Sclerotium rolfsii (Sacc) and Rhizoctonia bataticola (Taub). Arch. Phytopathol. Plant Prot. 2009, 42, 65-72. [CrossRef]

30. Kashkooli, A.B.; Saharkhiz, M.J. Essential Oil Compositions and Natural Herbicide Activity of Four Denaei Thyme (Thymus daenensis Celak.) Ecotypes. J. Essent. Oil-Bear. Plants 2014, 17, 859-874. [CrossRef]

31. Azirak, S.; Karaman, S. Allelopathic Effect of Some Essential Oils and Components on Germination of Weed Species. Acta Agric. Scand. Sect. B Soil Plant Sci. 2008, 58, 88-92. [CrossRef] 
32. Prajapati, V.; Tripathi, A.K.; Aggarwal, K.K.; Khanuja, S.P.S. Insecticidal, Repellent and Oviposition-Deterrent Activity of Selected Essential Oils against Anopheles stephensi, Aedes aegypti and Culex quinquefasciatus. Bioresour. Technol. 2005, 96, 1749-1757. [CrossRef]

33. Benelli, G.; Pavela, R.; Canale, A.; Cianfaglione, K.; Ciaschetti, G.; Conti, F.; Nicoletti, M.; Senthil-Nathan, S.; Mehlhorn, H.; Maggi, F. Acute Larvicidal Toxicity of Five Essential Oils (Pinus nigra, Hyssopus officinalis, Satureja montana, Aloysia citrodora and Pelargonium graveolens) against the Filariasis Vector Culex quinquefasciatus: Synergistic and Antagonistic Effects. Parasitol. Int. 2017, 66, 166-171. [CrossRef]

34. Park, I.K.; Choi, K.S.; Kim, D.H.; Choi, I.H.; Kim, L.S.; Bak, W.C.; Choi, J.W.; Shin, S.C. Fumigant Activity of Plant Essential Oils and Components from Horseradish (Armoracia rusticana), Anise (Pimpinella anisum) and Garlic (Allium sativum) Oils against Lycoriella ingenua (Diptera: Sciaridae). Pest Manag. Sci. 2006, 62, 723-728. [CrossRef]

35. Tu, X.F.; Hu, F.; Thakur, K.; Li, X.L.; Zhang, Y.S.; Wei, Z.J. Comparison of Antibacterial Effects and Fumigant Toxicity of Essential Oils Extracted from Different Plants. Ind. Crops Prod. 2018, 124, 192-200. [CrossRef]

36. Dhima, K.V.; Vasilakoglou, I.B.; Gatsis, T.D.; Panou-Pholotheou, E.; Eleftherohorinos, I.G. Effects of aromatic plants incorporated as green manure on weed and maize development. Field Crops Res. 2009, 110, 235-241. [CrossRef]

37. Mander, L.; Liu, H. Comprehensive Natural Products II: Chemistry and Biology Development Modification of Bioactivity; Elsevier: Oxford, UK, 2010; Volume 1.

38. Ibáñez, M.; Blázquez, M. Phytotoxicity of Essential Oils on Selected Weeds: Potential Hazard on Food Crops. Plants 2018, 7, 79. [CrossRef]

39. Wogiatzi, E.; Gougoulias, N.; Papachatzis, A.; Vagelas, I.; Chouliaras, N. Greek Oregano Essential Oils Production, Phytotoxicity and Antifungal Activity. Biotechnol. Biotechnol. Equip. 2009, 23, 1150-1152. [CrossRef]

40. Kordali, S.; Cakir, A.; Ozer, H.; Cakmakci, R.; Kesdek, M.; Mete, E. Antifungal, Phytotoxic and Insecticidal Properties of Essential Oil Isolated from Turkish Origanum acutidens and Its Three Components, Carvacrol, Thymol and p-Cymene. Bioresour. Technol. 2008, 99, 8788-8795. [CrossRef] [PubMed]

41. Elshafie, H.S.; Armentano, M.F.; Carmosino, M.; Bufo, S.A.; De Feo, V.; Camele, I. Cytotoxic Activity of Origanum vulgare L. on Hepatocellular carcinoma Cell Line HepG2 and Evaluation of Its Biological Activity. Molecules 2017, 22, 1435. [CrossRef] [PubMed]

42. Saharkhiz, M.J.; Smaeili, S.; Merikhi, M. Essential Oil Analysis and Phytotoxic Activity of Two Ecotypes of Zataria multiflora Boiss. Growing in Iran. Nat. Prod. Res. 2010, 24, 1598-1609. [CrossRef] [PubMed]

43. Amini, S.; Azizi, M.; Joharchi, M.R.; Moradinezhad, F. Evaluation of Allelopathic Activity of 68 Medicinal and Wild Plant Species of Iran by Sandwich Method. Int. J. Hortic. Sci. Technol. 2016, 3, 243-253. [CrossRef]

44. Mohammadi, A.; Hashemi, M.; Hosseini, S.M. Comparison of Antifungal Activities of Various Essential Oils on the Phytophthora drechsleri, the Causal Agent of Fruit Decay. Iran. J. Microbiol. 2015, 7, 31-37.

45. Vivanco, J.M.; Paschke, M.W.; Callaway, R. Allelochemical Control of Non-Indigenous Invasive Plant Species Affecting Military Testing and Training Activities, Final Report, U.S. Department of Defense Strategic Environmental Research and Development Program (SERDP) Project RC-1388. 2010. [CrossRef]

46. Abad, M.J.; Bedoya, L.M.; Apaza, L.; Bermejo, P. The Artemisia L. Genus: A Review of Bioactive Essential Oils. Molecules 2012, 17, 2542-2566. [CrossRef]

47. Chowhan, N.; Singh, H.P.; Batish, D.R.; Kohli, R.K. Phytotoxic Effects of $\beta$-Pinene on Early Growth and Associated Biochemical Changes in Rice. Acta Physiol. Plant. 2011, 33, 2369-2376. [CrossRef]

48. Nerio, L.S.; Olivero-Verbel, J.; Stashenko, E. Repellent Activity of Essential Oils: A Review. Bioresour. Technol. 2010, 101, 372-378. [CrossRef]

49. Ibrahim, M.A.; Kainulainen, P.; Aflatuni, A.; Tiilikkala, K.; Holopainen, J.K. Insecticidal, Repellent, Antimicrobial Activity and Phytotoxicity of Essential Oils: With Special Reference to Limonene and Its Suitability for Control of Insect Pests. Agric. Food Sci. Finland 2001, 10, 243-259. [CrossRef]

50. Sfara, V.; Zerba, E.N.; Alzogaray, R.A. Fumigant Insecticidal Activity and Repellent Effect of Five Essential Oils and Seven Monoterpenes on First-Instar Nymphs of Rhodnius prolixus. J. Med. Entomol. 2009, 46, 511-515. [CrossRef] [PubMed]

51. Klocke, J.A.; Darlington, M.V.; Balandrin, M.F. 1,8-Cineole (Eucalyptol), a Mosquito Feeding and Ovipositional Repellent from Volatile Oil of Hemizonia fitchii (Asteraceae). J. Chem. Ecol. 1987, 13, 2131-2141. [CrossRef] [PubMed] 
52. Yoneyama, K.; Natsume, M. Allelochemicals for Plant-Plant and Plant-Microbe Interactions; Elsevier Inc.: Philadelphia, PA, USA, 2010. [CrossRef]

53. Alonso-Amelot, M.E. Multitargeted Bioactive Materials of Plants in the Curcuma Genus and Related Compounds: Recent Advances. In Studies in Natural Products Chemistry; Elsevier B.V.: Amsterdam, The Netherlands, 2016; Volume 47, pp. 111-200. [CrossRef]

54. Fischer, N.H.; Williamson, G.B.; Tanrisever, N.; de la Pena, A.; Weidenhamer, J.D.; Jordan, E.D.; Richardson, D.R. Allelopathic Actions in the Florida Scrub Community. Biol. Plant. 1989, 31, 471-478. [CrossRef]

55. Hossain, F.; Follett, P.; Dang Vu, K.; Harich, M.; Salmieri, S.; Lacroix, M. Evidence for Synergistic Activity of Plant-Derived Essential Oils against Fungal Pathogens of Food. Food Microbiol. 2016, 53, 24-30. [CrossRef] [PubMed]

(C) 2020 by the authors. Licensee MDPI, Basel, Switzerland. This article is an open access article distributed under the terms and conditions of the Creative Commons Attribution (CC BY) license (http://creativecommons.org/licenses/by/4.0/). 